OPEN ACCESS

Edited by:

Richard Ribon Fletcher, Massachusetts Institute of Technology, United States

Reviewed by: Aline Paes,

Universidade Federal Fluminense,

Brazil

Martin Gerbert Frasch,

Department of Obstetrics and Gynecology, School of Medicine,

University of Washington United States

Paolo Melillo,

University of Campania Luigi Vanvitelli,

Maria G. Signorini,

Politecnico di Milano, Italy

*Correspondence:

Camilo E. Valderrama

cvalder@emory.edu

Gari D. Clifford

gari@gatech.edu

Specialty section

This article was submitted to Machine Learning and Artificial Intelligence,

a section of the journal Frontiers in Artificial Intelligence

Received: 21 November 2019

Accepted: 29 June 2020

Published: 07 August 2020

Citation:

Valderrama CE, Marzbanrad $F$ Hall-Clifford $R$, Rohloff $P$ and

Clifford GD (2020) A Proxy for Detecting IUGR Based on Gestational Age Estimation in a Guatemalan Rural

Population. Front. Artif. Intell. 3:56. doi: 10.3389/frai.2020.00056

\section{A Proxy for Detecting IUGR Based on Gestational Age Estimation in a Guatemalan Rural Population}

\author{
Camilo E. Valderrama ${ }^{1 *}$, Faezeh Marzbanrad ${ }^{2}$, Rachel Hall-Clifford ${ }^{3}$, Peter Rohloff ${ }^{4,5}$ and \\ Gari D. Clifford ${ }^{1,6 *}$ \\ ${ }^{1}$ Department of Biomedical Informatics, Emory University, Atlanta, GA, United States, ${ }^{2}$ Department of Electrical and \\ Computer Systems Engineering, Monash University, Melbourne, VIC, Australia, ${ }^{3}$ Department of Sociology, Center for the \\ Study of Human Health, Emory University, Atlanta, GA, United States, ' Wuqu' Kawoq I Maya Health Alliance, \\ Santiago Sacatepéquez, Guatemala, ${ }^{5}$ Division of Global Health Equity, Brigham and Women's Hospital, Boston, MA, \\ United States, ${ }^{6}$ Department of Biomedical Engineering, Georgia Institute of Technology, Emory University, Atlanta, GA, \\ United States
}

In-utero progress of fetal development is normally assessed through manual measurements taken from ultrasound images, requiring relatively expensive equipment and well-trained personnel. Such monitoring is therefore unavailable in low- and middle-income countries (LMICs), where most of the perinatal mortality and morbidity exists. The work presented here attempts to identify a proxy for IUGR, which is a significant contributor to perinatal death in LMICs, by determining gestational age (GA) from data derived from simple-to-use, low-cost one-dimensional Doppler ultrasound (1D-DUS) and blood pressure devices. A total of 114 paired 1D-DUS recordings and maternal blood pressure recordings were selected, based on previously described signal quality measures. The average length of 1D-DUS recording was $10.43 \pm 1.41 \mathrm{~min}$. The $\mathrm{min} / \mathrm{median} / \mathrm{max}$ systolic and diastolic maternal blood pressures were 79/102/121 and $50.5 / 63.5 / 78.5 \mathrm{mmHg}$, respectively. GA was estimated using features derived from the 1D-DUS and maternal blood pressure using a support vector regression (SVR) approach and GA based on the last menstrual period as a reference target. A total of 50 trials of 5-fold cross-validation were performed for feature selection. The final SVR model was retrained on the training data and then tested on a held-out set comprising 28 normal weight and 25 low birth weight (LBW) newborns. The mean absolute GA error with respect to the last menstrual period was found to be 0.72 and 1.01 months for the normal and LBW newborns, respectively. The mean error in the GA estimate was shown to be negatively correlated with the birth weight. Thus, if the estimated GA is lower than the (remembered) GA calculated from last menstruation, then this could be interpreted as a potential sign of IUGR associated with LBW, and referral and intervention may be necessary. The assessment system may, therefore, have an immediate impact if coupled with suitable intervention, such as nutritional supplementation. However, a prospective clinical trial is required to show the efficacy of such a metric in the detection of IUGR and the impact of the intervention.

Keywords: gestational age estimation, one-dimension Doppler ultrasound (1D-DUS), fetal heart rate (FHR), intra-uterine growth restriction (IUGR), maternal blood pressure, signal processing, supervised machine learning, low-and middle-income countries (LMICs) 


\section{INTRODUCTION}

Estimation of fetal gestational age (GA) provides important information throughout pregnancy, such as delivery scheduling, growth disorder detection, and preterm newborns management (Alexander et al., 1995). Thus, GA estimation can assist in detecting issues leading to perinatal mortality and morbidity (Rijken et al., 2014; Karl et al., 2015). This detection is particularly needed in low-and middle-income countries (LMICs), which account for $\sim 98 \%$ of all reported perinatal deaths worldwide, largely due to gestational developmental issues (Zupan, 2005).

In high-income countries, clinical teams generally use ultrasound images to estimate GA, as well as any structural abnormalities (Malhotra et al., 2014). These GA estimations are based on a variety of fetal measurements, such as biparietal diameter, crown-rump length, head circumference, abdominal circumference, and femur length (Malhotra et al., 2014). However, in LMICs, the access to ultrasound imaging is limited, and almost unavailable in rural areas, due to the high cost of the medical equipment, the expenses for maintenance, and the requirement of skilled medical staff (World Health Organization, 2014). Hence, low-cost alternative methods for dating gestation are used in LMICs.

A common low-cost method used for GA estimation is the last menstrual period (LMP), in which a 28-days menstrual cycle is assumed. Although previous studies have criticized LMP due to the inconsistency in the menstrual cycle length (Dietz et al., 2007), and the difficulty to recall the day of the last menstrual period (Andersen et al., 1981), the LMP method has shown to be a somewhat useful method for LMICs, particularly in rural areas lacking medical equipment. In fact, Neufeld et al. (2006) compared 171 GA estimations based on LMP collected in rural Guatemala with GA estimations given by biparietal diameter, reporting that GA estimations by the LMP were within \pm 14 days of the biparietal diameter estimations for $94 \%$ of the cases.

GA estimations based on LMP can assist in the assessment of intrauterine growth restriction (IUGR), which has a prevalence varying between 9 and $11 \%$ in LMICs (de Onis et al., 1998; Lee et al., 2013). Specifically, IUGR is assessed by comparing the estimate of GA with the symphysis-fundal height measurement (World Health Organization, 2016). For fetuses growing

\footnotetext{
Abbreviations: AAC, acceleration average capacity; ADASYN, adaptive synthetic sampling; ANS, autonomic nervous system; ApEn, approximate entropy; AUROC, area under the receiver operating characteristic; BPM, beat per minute; $\mathrm{CO}$, cardiac output; DAC, deceleration average capacity; DBP, diastolic blood pressure; DUS, Doppler ultrasound signal; FHR, fetal heart rate; fHRV, fetal heart rate variability; GA, gestational age; GBT, gradient boosting tree; GMI, generalized mutual information; HF, high frequency $(0.5-1 \mathrm{~Hz})$; II, interval index; IUGR, intrauterine growth restriction; LBW, low birth weight; LF, low frequency $(0.03-0.15 \mathrm{~Hz})$; LMICs, low- and middle-income countries; LMP, last menstrual period; LTV, long term variability; MAE, mean absolute error; MAP, mean arterial pressure; MF, medium frequency $(0.15-0.5 \mathrm{~Hz})$; mIS, mean of the interbeat sequence; MHR, maternal heart rate; mRMR, minimum redundancy and maximum relevance algorithm; MSI, modified shock index; NBW, normal birth weight; PNN5, percentage of consecutive beats that differ by more than $5 \mathrm{~ms}$; PP, pulse pressure; rmssdIs, root mean square of successive differences; RPP, rate pressure product; SBP, systolic blood pressure; SI, shock index; stdIS, standard deviation of the interbeat sequence; STV, short term variability; SV, stroke volume; SVR, support vector regression; varIS, variance of the interbeat sequence.
}

normally, from 24 weeks of gestation, the symphysis-fundal height measurement $\left(L_{s f h}\right)$ in centimeters should correspond to the number of weeks of gestation $\pm 2 \mathrm{~cm}$. When $L_{s f h}<N-$ 2 , where $N$ is the number of weeks since the last menstrual period, the fetus is suspected to be IUGR (Peter et al., 2015). However, the symphysis-fundal height method lacks significant evidence to recommend its widely use in LMICs (World Health Organization, 2016). Moreover, previous studies have noted that the SHF has exhibited a large error of \pm 6 weeks for estimating GA (Griffiths et al., 2008). New approaches are, therefore, still needed to provide reproducible and low-cost assessment for detecting abnormal growth in settings in which ultrasound images, taken by trained operators, are not available.

In this work, we propose an alternative approach for GA estimation to provide a proxy for assessing fetal development and identifying possible cases of IUGR for a Guatemalan rural population, in which ultrasound imaging is not affordable and the symphysis-fundal height is not accurate. Our approach estimates GA using fetal heart rate variability (fHRV) indexes and maternal hemodynamics derived from one-dimensional Doppler ultrasound (1D-DUS) and maternal blood pressure, respectively. Data were acquired during routine perinatal check-up visits by traditional birth attendants using a low-cost Doppler transducer and a self-inflating blood pressure device (Stroux et al., 2016; Martinez et al., 2017, 2018). These features were used to build a machine learning algorithm to estimate GA. We hypothesized that if the estimated GA is lower than the GA calculated from last menstruation, then this could be interpreted as a potential sign of IUGR based on low birth weight (LBW), and referral and intervention may be necessary.

\section{BACKGROUND}

Fetal heart rate is influenced by the Autonomic Nervous System (ANS) (Schneider et al., 2009; Wallwitz et al., 2012), which in turn modifies FHR dynamics over the course of pregnancy. In particular, FHR variability evolves over the course of pregnancy and may reflect the maturity of the ANS, and thus may indicate the fetal GA. Wakai (2004) reported that fHRV, as observed from traces taken from 61 pregnant women without complications, increases during gestation. In particular, they noted that short term variability increased during the last trimester, whereas long term variability exhibited the largest increases in the early gestational period. Figure 1 shows an example of how FHR changes across gestation, as reported in Wakai (2004).

Based on FHR, previous studies have shown a correlation between GA and markers derived from fHRV. Linear metrics, such as the mean of R-R interval, the standard deviation and root mean square of successive differences positively correlated with GA for both genders (Lange et al., 2005). Non-linear metrics, such as approximate entropy (ApEn), Lyapunov exponent, toneentropy and generalized mutual information, have also been linked to fetal maturation (Van Leeuwen et al., 1999; Hoyer et al., 2012; Khandoker et al., 2015). Additionally, Van Leeuwen et al. (2003) and Signorini et al. (2003) reported that power in the $0.003-1.0 \mathrm{~Hz}$ frequency band vary during pregnancy. 


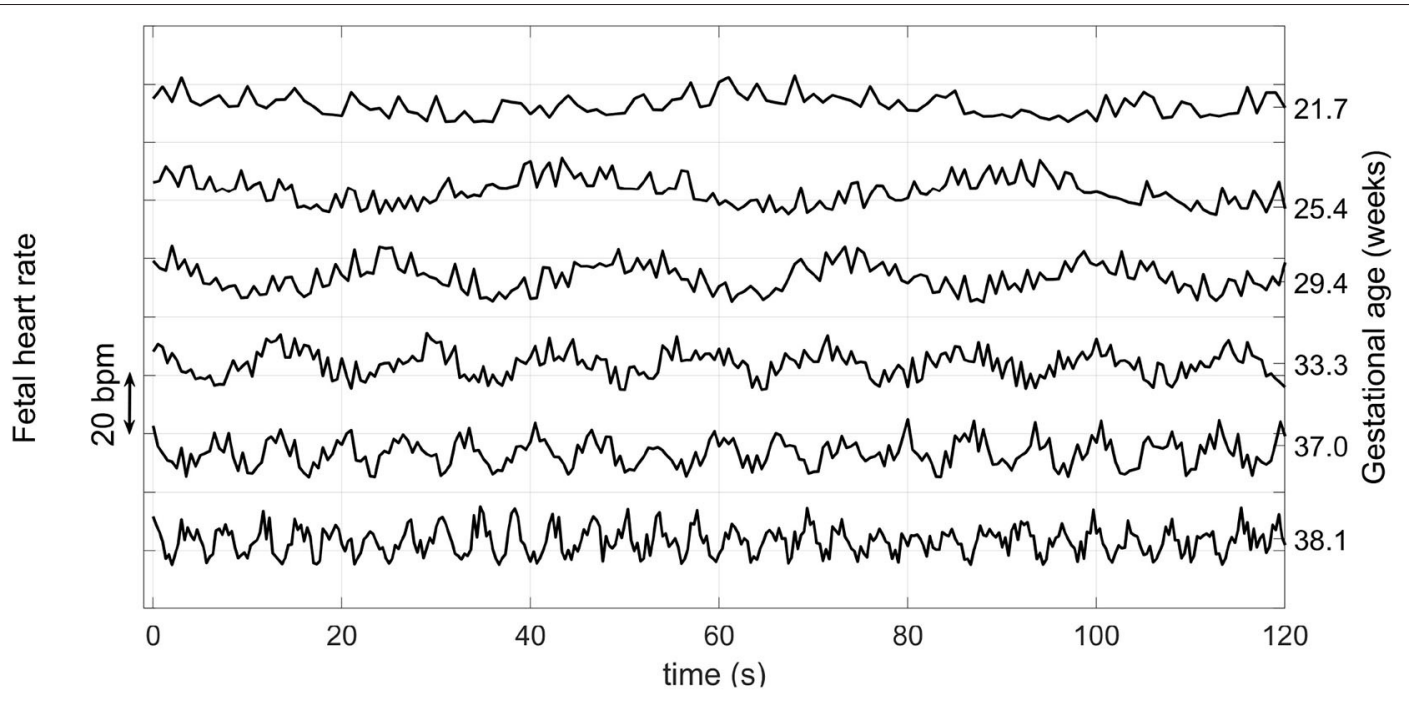

FIGURE 1 | Variation of the FHR from 22 to 38 weeks during pregnancy. Note that the vertical axis has an arbitrary offset. Average FHR does not drop by such a large amount each week during pregnancy, but rather it drops on average by about 15 BPM from week 25 to 40 (Kapaya et al., 2018). Adapted from Wakai (2004).

Initial works on GA estimation aimed to find a relation between GA and FHR-based metrics using univariate regression (Hoyer et al., 2012). More recently, some works have aimed to improve the characterizing of FHR by incorporating multivariate and more complex methods. In particular, Tetschke et al. (2016) extracted features from 359 high resolution fetal magnetocardiographic recordings, lasting at least $20 \mathrm{~min}$. The researchers implemented an algorithm to extract non-active portions of the recording and calculated both linear and non-linear metrics of fHRV from the these quiet periods. Results showed that entropy and skewness were more highly correlated with GA than those obtained by traditional linear HRV metrics. However, this approach requires high temporal and spatial resolution data acquired from costly and non-portable equipment, making its use in LMICs impractical.

In earlier work, Marzbanrad et al. (2016) estimated GA for 57 fetuses using a step-wise regression based on cardiac wall intervals derived from one-dimension Doppler ultrasound signal (1D-DUS) and fECG signals recorded in a Japanese hospital. The estimated GAs were compared to the GA derived from crown-rump length, achieving a mean square error of 3.8 and 5.1 weeks for cardiac intervals and fHRV parameters, respectively. In further work, Marzbanrad et al. (2017) improved the estimation accuracy by incorporating 1D-DUS and fECG quality assessment algorithms to filter poor quality signals. As a result, the stepwise regression achieved a mean absolute error (MAE) of 4.7 weeks from fHRV parameters, and 2.7 weeks when including the cardiac intervals metrics. Although this latter method achieved comparable results to Doppler imaging based estimations, it required two sources, $1 \mathrm{D}$-DUS and fECG signals, which increases costs and complicates implementation, particularly in LMICs (Stroux et al., 2016).

In addition to FHR indexes, maternal blood pressure is also a relevant metric for GA estimation. Previous works have reported that maternal systolic and diastolic blood pressure increases throughout pregnancy (Steer et al., 2004; Salas et al., 2006; Kac et al., 2015; Rebelo et al., 2015). However, despite the correlation between GA and maternal blood pressure, no research has included maternal blood pressure in regression models to estimate GA. We note that extreme blood pressures may be indicative of pre-eclampsia, or other gestational issues. It is therefore important to treat these separately.

\section{METHODS}

\subsection{Databases}

\subsubsection{Collection of the Data}

Data used in this work were collected as a part of a randomized control trial conducted in rural highland Guatemala in the vicinity of Tecpan, Chimaltenango. This program was approved by the Institutional Review Boards of Emory University, the Wuqu' Kawoq | Maya Health Alliance, and Agnes Scott College (Ref: IRB00076231- "Mobile Health Intervention to Improve Perinatal Continuum of Care in Guatemala") and registered on ClinicalTrials.gov (identifier NCT02348840). In the trial, traditional birth attendants were trained to use a mobile mHealth system to record perinatal information during approximately monthly visits during the second and third trimesters. More details on the design and implementation of the mobile mHealth system, and the training of the traditional birth attendants can be found in Stroux et al. (2016) and Martinez et al. (2017, 2018). At this time, the dataset is not publicly available; however, a deidentified dataset can be available upon request and approbation of the project ethical committee.

The perinatal care program included both prenatal and post-partum visits. In the prenatal visits, traditional birth attendants recorded GA in months by counting the number of whole months since the last menstrual period. The GA was recorded in months instead of weeks to reduce measurement errors since usually patients attended in this project forgot 
the specific date of their last menstrual period, and very few received an early obstetrical ultrasound for more accurate dating (Martinez et al., 2017). During the visit, the traditional birth attendants also recorded 1D-DUS signals and blood pressure from the mother in a supine position using the mobile mHealth system (Stroux et al., 2016; Martinez et al., 2017, 2018). The 1D-DUS signals were recorded using a Doppler transducer (AngelSounds Fetal Doppler JPD-100s, Jumper Medical Co., Ltd., Shenzhen, China) with an ultrasound transmission frequency of $3.3 \mathrm{MHz}$ and a digitization sampling frequency of 44.1 $\mathrm{kHz}$. The maternal blood pressure was taken from both arms using a self-inflating blood pressure device calibrated for pregnancy.

In the post-partum visits, traditional birth attendants recorded the newborn's birth date, sex, current weight, length, and head size. These post-partum visits could occur days or months after birth since sometimes it was difficult to follow up on the patients. The birth weight was then estimated using a Reed 2 second-order model (Berkey and Reed, 1987) fitted on 917 observed postnatal weights using an approach we have previously described in Valderrama et al. (2020). A weight threshold was used to classify the estimated birth weights as low or normal. This threshold was defined by first finding the percentile corresponding to $\leq 2.5 \mathrm{~kg}$ in a Guatemalan national maternal survey for the region of relevance in our study (MSPAS/Guatemala et al., 2017). We found that the lowest $14.3 \%$ of male newborns and $16.33 \%$ of female newborns satisfied this weight criterion. These percentiles were then located in our estimated birth weight distribution to determine the corresponding LBW threshold. This corresponded to $2.64 \mathrm{~kg}$ for males and $2.57 \mathrm{~kg}$ for females.

\subsubsection{Assumption of the Study}

In this work, a newborn was considered as a possible case of IUGR if their estimated birth weight was below the threshold discussed above. This assumption is based on the fact that LBW could be a consequence of either preterm birth ( $<37$ weeks) or small-for-gestational-age. However, in LMICs, around $60 \%$ of LBW newborns are small-for-gestational-age (Lee et al., 2013), and the main reason for small-for-gestational-age in LMICs is IUGR (de Onis et al., 1998; Lee et al., 2013).

\subsubsection{Data Inclusion Criteria}

Prenatal visits were included if they contained both blood pressure pictures and 1D-DUS recordings with some conditions. For the blood pressure, the numbers had to be readable on the photograph of the blood pressure device. Also, the difference between the right and left arm measurements had to be lower than $15 \mathrm{mmHg}$, thus discarding any spurious measurements. Finally, possible preeclampsia patients were discarded when systolic or diastolic blood pressure was higher than a threshold. This threshold was defined at 130 and $80 \mathrm{mmHg}$ for SBP and $\mathrm{DBP}$, as is suggested for measurements taken in spine position (Netea et al., 2003; Kluttig et al., 2010; Cicolini et al., 2011).

The conditions for including the 1D-DUS recording were based on length and quality. The minimum length was fixed at $10 \mathrm{~min}$ since earlier work suggests that this is the required length to extract fHRV indexes, such as baseline, accelerations, and decelerations (Dobbe et al., 2001).

In addition to the length, the quality of a $1 \mathrm{D}$-DUS recording was also considered as an inclusion criterion. The 1D-DUS quality was assessed using a window of $3.75 \mathrm{~s}$ and a sliding window of $250 \mathrm{~ms}$. For each 3.75-s window, 16 features were extracted, including Wavelet percentage energy in the range 250-2,000 Hz, Mel-frequency cepstral coefficients, and power spectrum ratios on electrical interference frequency ranges. The features were fed into a classifier composed of a logistic regression and a multiclass support vector machine to classify the 3.75-s window into good quality, interference, silence, talking in the background, or low signal to noise ratio. More details of the quality assessment method can be found in Valderrama et al. (2017, 2018a).

Based on the length and quality criteria, a 1D-DUS recording was only included in this present work if it lasted more than $10 \mathrm{~min}$, and at least $50 \%$ of its 3.75 -s windows were labeled as good quality.

\subsubsection{Final Data Set}

After applying the inclusion criteria, the final dataset comprised 167 visits from 153 non-preeclampsia women who were pregnant with singleton fetuses. From these patients, 142 gave birth to normal weight singletons, whereas 24 gave birth to LBW newborns, based on our thresholds defined for the study population (Valderrama et al., 2020) (see subsection 3.1.1).

Table 1 shows demographics of the patients. The male/female ratio was higher in the LBW group than in the normal birth weight group. On the other hand, the maternal age and the number of previous pregnancies (gravidity) were higher in the normal weight group.

Table 2 shows the distribution of the GA based on the last menstrual period (LMP) method. Visits ranged from the sixth to the ninth month of pregnancy, focusing mainly on the third trimester.

\subsection{Approach Overview}

Figure 2 shows an overview of the approach used to detect possible cases of IUGR. First, 37 features were extracted from the raw $1 \mathrm{D}$-DUS signals and maternal blood pressure readings. The extracted dataset was split into three subsets: normal birth weight (NBW) training set, NBW test set, and LBW test set. The training set was used to select the best regression model and features. The

TABLE 1 | Average demographics for the data used in this study.

\begin{tabular}{lcc}
\hline Variable & Normal birth weight & Low birth weight \\
\hline Patients (count) & 129 & 24 \\
Newborn gender (male/female) & $56 / 73$ & $17 / 7$ \\
Birth weight (kg) & $3.1(S D=0.3 ; N=129)$ & $2.3(S D=0.4 ; N=24)$ \\
Maternal age (years) & $27.0(S D=6.3 ; N=123) 24.5(S D=6.7 ; N=22)$ \\
Gravidity (count) & $3.4(S D=2.5 ; N=96)$ & $2.3(S D=3.1 ; N=16)$
\end{tabular}

For each metric, the standard deviation and the number of patients available for that variable are shown in parenthesis. 
selected regression model and features were used to estimate GA from the NBW and LBW held-out test sets. The GA estimate errors of the NBW and LBW were compared to assess if birth weight affected the GA estimation. The following subsections provide details of each component of the approach.

\subsection{Deriving the FHR Signal}

\subsubsection{Extracting Fetal Heart Rate From 1D-DUS}

Each 1D-DUS recording was analyzed using a window of 3.75 $\mathrm{s}$ and a sliding window of $0.25 \mathrm{~s}$. The window length was set at $3.75 \mathrm{~s}$ since it is the usual length for computerized analysis of fetal non-stress tests based on the Dawes/Redman criteria (Dawes et al., 1981; Pardey et al., 2002). The selection of the sliding window was based on the desired sampling frequency, namely $4 \mathrm{~Hz}$. This sampling frequency has been shown to be sufficient for digital cardiotocography (Romagnoli et al., 2019),

TABLE 2 | Number of visits per gestational age (GA) taken with the last menstrual period (LMP) method.

\begin{tabular}{lcc}
\hline Gestational age (months) & Normal weight & Low birth weight \\
\hline 6 & 9 & 3 \\
7 & 38 & 7 \\
8 & 37 & 5 \\
9 & 58 & 10 \\
\hline Total & 142 & 25
\end{tabular}

and corresponded to a Nyquist frequency of $2 \mathrm{~Hz}$, thus allowing the extraction of spectral metrics in the range $0.03-1 \mathrm{~Hz}$.

For each 3.75-s window, the fetal heart rate (FHR) was estimated auto-correlation (AC)-based method using an open source code written in Matlab (MathWorks, Natick, MA, USA), previously introduced in (Valderrama et al., 2018b, 2019). Specifically, the method detects the fundamental period of the envelope of the 3.75-s window by applying auto-correlation, and then the FHR is estimated by dividing 60 between the fundamental period in seconds. More details of the FHR estimator are found in (Valderrama et al., 2019).

In addition to estimate the FHR, the quality of the 3.75-s window was also assessed and stored for further prepossessing steps. The quality was assessed using the method presented in Valderrama et al. (2018a) (see subsection 3.1.3).

\subsubsection{Pre-processing of Estimated FHR Signal}

Since 1D-DUS recordings are prone to noise, any given 3.75-s window of Doppler data may lead to an unreliable estimate. Two steps then assessed the reliability of the extracted FHR. Firstly, as recommended in Nyboe (2011), we removed FHR estimates that were not within the $65-175 \%$ range of the average of the previous two estimates. Secondly, we removed 3.75-s windows classified as something else other than good quality.

Each discarded value was replaced by the linear interpolation between its previous point and the next stable segment. A stable segment was defined as a region of five adjacent points for which the FHR estimate did not vary by more than ten beat per minute (BPM).

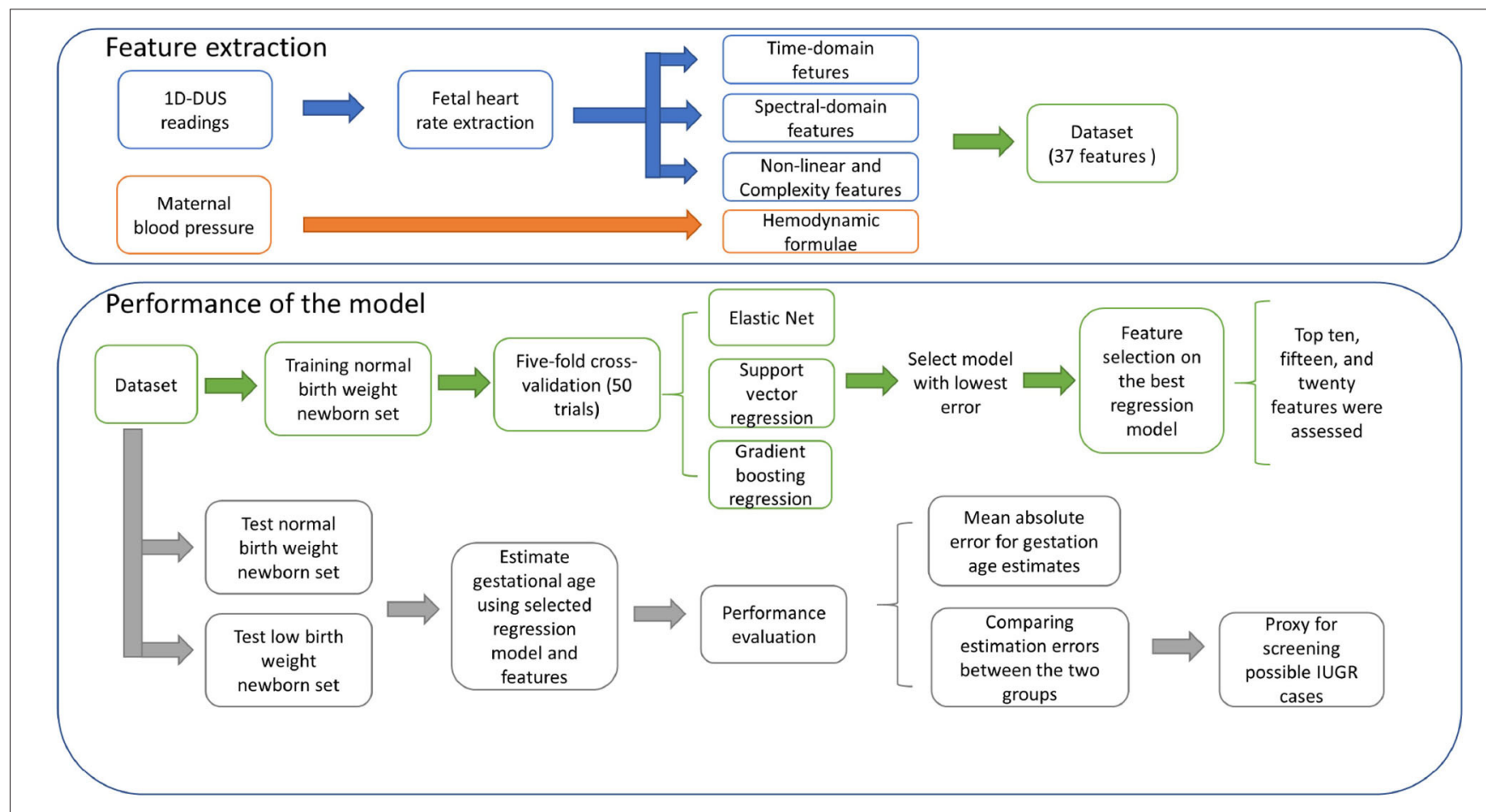

FIGURE 2 | Block diagram of the approach for detecting possible cases of IUGR. 


\subsubsection{Calculation of the Baseline, Acceleration, and Deceleration}

The baseline was determined using an algorithm proposed by Andersson (2011), which is an improvement of those proposed by Dawes et al. (1982) and Mantel et al. (1990). Specifically, a filter bank was applied to the $4-\mathrm{Hz}$ FHR time series to attenuate any accelerations or deceleration.

Following the work of Andersson (2011), accelerations and decelerations were detected for each valid 1-min segment of the baseline. A valid segment was determined by computing a FHR histogram using a bin width of 10 BPM. If the most frequent bin of the histogram contained more than $40 \%$ of the values, the 1-min baseline segment was considered valid.

When a 1-min baseline segment was determined to be valid, acceleration and deceleration intervals were identified following Dawes criteria (Dawes et al., 1982). Namely, an acceleration was defined to be a section of data for which the FHR was higher than the baseline for at least $15 s$ and at least one sample was 15 BPM or more above the baseline. Similarly, a deceleration was defined where the FHR remained below the baseline FHR for at least 15 s, and at least one sample was 15 BPM below the baseline. More details of the algorithm can be found in Andersson (2011).

\subsection{Features Used for Gestational Age Estimation}

Based on previous works presented in section 2, a total of 37 features relevant for estimating GA were extracted from the 1DDUS and blood pressure device captured at the perinatal visits.

\subsubsection{Features Extracted From the 1D-DUS}

The features derived from 1D-DUS recording were calculated using the FHR time series (see section 3.3). Since the RR-interval sequence is necessary to estimate fHRV metrics, the FHR series was converted into a interbeat sequence as:

$$
T(i)=60,000 / S(i),
$$

where $S(i)$ is the FHR at the $i-t$ second.

Table 3 shows the total features extracted from the FHR time series and the interbeat sequence. The table also provides the physiological interpretation for each feature and relevant previous works in which such features have been used to assess gestational development. The features were grouped into three categories: linear time-domain, non-linear and complexity, and frequency-domain features.

\subsubsection{Features Generated From Maternal Blood Pressure Readings}

The maternal systolic blood pressure (SBP), diastolic blood pressure (DBP), and the maternal heart rate (MHR) measurements from the blood pressure device were used as features. Since the SBP, DBP, and MHR were taken for both patient's arms, these values were averaged. The averaged SBP, DBP, MHR were used to generate seven additional hemodynamics formulas, which have been reported to vary throughout pregnancy (Steer et al., 2004; Salas et al., 2006; Kac et al., 2015; Rebelo et al., 2015). Table 4 listed all the features generated from maternal blood pressure readings.

\subsection{Assessing Potential of Extracted Features}

To evaluate the potential of fetal cardiac and maternal blood pressure features to describe the fetal development throughout pregnancy, the Pearson correlation coefficient, and its $p$-value, was calculated for each feature and the gestational age. This rationale was based on previous research articles that have reported linear relationships between cardiac physiological parameters and fetal maturity (Frasch et al., 2007; Hoyer et al., 2019; Signorini et al., 2020). Thus, the correlation coefficients help to check that features used in this work are relevant for GA estimation.

\subsection{Estimation of Gestational Age}

All of the features were extracted for both the 129 normal birth weight and 24 LBW newborns at each stage of pregnancy for which data was available. The GA estimation model was training only with visits of newborns with normal birth weights because previous research has reported that LBW fetuses have discrepancies in their GA estimations from fHRV (Marzbanrad et al., 2017). However, the features derived from the recordings of the LBW newborns were used later to test the model's ability to estimate GA.

The 129 normal weight patients were split into training and test sets. The number of patients for the test set was selected to be proportional to the LBW newborn set. A Wilcoxon rank-sum hypothesis test (two-sided; $\alpha=0.05$ ) was applied in order to test whether there were statistically significant differences between the training and test sets for all the values of the 37 features (if a statistically significant difference was found, the subjects were randomized again).

The training set comprised 104 newborns, from which 95 had one visit, eight had two visits, and one had three visits, giving a total of 114 visits. For the test set there were a total of 25 normal birth weight newborns, for which 22 had one visit and three had two visits, giving a total of 28 visits. Table 5 shows the number of visits for each GA for the training and test sets.

\subsubsection{Training/Validation Methodology}

The training and validation procedure was performed using a 5fold cross validation with 50 trials (repetitions). At each trial, the patients were randomly assigned to different folds, ensuring that visit features corresponding to the same patient were in the same folder. Thus, at each iteration, $\sim 20 \%$ of the training data was used for validation (held-out fold). By using 50 trials the variability of the models for estimating the GA could be measured, and confidence intervals could be estimated.

Since folds were class unbalanced (i.e., different number of visit for each gestational age), at each iteration of the 5-fold cross-validation, the number of visits per GA was balanced on the training folds before constructing a model. To that end, we used the Adaptive Synthetic Sampling (ADASYN) method, which has been reported to overcome the class imbalance problem in support vector machine models (Batuwita and Paladey, 2013). This method generates synthetic data for the minority classes by taking the Euclidean distance between two data points and then adding the difference scaled by a factor, 
TABLE 3 | Features extracted from 1D-DUS signals.

\begin{tabular}{|c|c|}
\hline Feature & Calculation \\
\hline \multicolumn{2}{|c|}{ LINEAR TIME-DOMAIN FEATURES } \\
\hline $\begin{array}{l}\text { Short-term variability } \\
\text { (STV) }\end{array}$ & $\begin{array}{l}S T V_{j}=\frac{\sum_{i=2}^{24}\left|T_{j}(i)-T_{j}(i-1)\right|}{23}, \text { where } T_{j}(i) \text { is the } i-t h \\
\text { sample of the } j-\text { th minute of the interbeat sequence }\end{array}$ \\
\hline Interval Index (II) & $\begin{array}{l}\|_{j}=\frac{s t d\left\{\left|d T_{2}\right|, \ldots,\left|d T_{24}\right|\right\}}{S T V_{j}} \text {, where } s t d \text { is the standard } \\
\text { deviation of the sequence composed of the absolute } \\
\text { difference of successive samples } T_{j}(i)-T_{j}(i-1)\left(\left|d T_{i}\right|\right) \\
\text { of the } j-\text { th minute of the interbeat sequence }\end{array}$ \\
\hline $\begin{array}{l}\text { Long-term variability } \\
\text { (LTV) }\end{array}$ & $\begin{array}{l}L T V_{j}=\max \left[T_{j}\right]-\min \left[T_{j}\right], \text { where } T_{j} \text { is the } j-\text { th minute } \\
\text { of the interbeat sequence }\end{array}$ \\
\hline Long-term irregularity & $\mathrm{LTI}$ is defined as the interquartile range of the following \\
\hline$(\mathrm{LTI})$ & distribution: $m_{k}=\sqrt{\sum_{i=2}^{72} T_{k}^{2}(i)+T_{k}^{2}(i-1)}$, where $T_{k}(i)$ is \\
\hline
\end{tabular}

\section{STV/LTV}

Basal fetal heart rate

Number of accelerations per minute

Acceleration average capacity (AAC)

Deceleration average capacity (DAC)

\section{Mean (mls) \\ Standard deviation (stdls)}

Variance (varlS)

Root mean square of successive differences (rmssdIS)

rmssdIS/stdls

Skewness (skewnessiS) Kurtosis (kurtosisIS)

Variance of the interbeat-sequence

Root mean square of successive differences of the interbeat sequence

Ration between rmssdIS and stdls

Skewness of the interbeat-sequence

Kurtosis of the interbeat-sequence

\section{PNN5 The fraction of consecutive beats that differ by more than $5 \mathrm{~ms}$}

\section{NON-LINEAR AND COMPLEXITY FEATURES}

Approximate entropy (ApEn)
Interpretation

Relevant previous works
Variability within 1-min interval due to acceleration and decelarations

Fluctuation between variability of successive beat intervals and STV

Range of the interbeat sequence due to accelaration and decelarations (slow oscillations) Variability over 3 min intervals (Slow oscillations over longer time scales)
Lunghi et al., 2005; Fanelli 2014

Lunghi et al., 2005; Fanelli et al., 2013; Signorini et al., 2014

Lunghi et al., 2005; Fanelli et al., 2013

Signorini et al., 2014 et al., 2013; Signorini et al.,
Fluctuation between short and long variation of the interbeat sequence

Maturity of ANS; as pregnancy advances, the parasympathetic system matures and the FHR is reduced

Increment of accelerations due to maturity of vagal functions

Occurrence or absence of the appearance of accelerations

Occurrence or absence of the appearance of decelerations

Maturity of the parasympathetic system

Dispersion of the interbeat sequence. Variability among interbeat sequence increased as pregnancy progress due to accelerations and decelerations controlled by vagal function Variability among interbeat sequence

Variability between successive beat intervals due to accelerations and decelerations controlled by vagal function

Fluctuation between variability of successive beat intervals and variability of all the beat intervals Asymmetry on the FHR trace due to accelerations and decelerations controlled by vagal function

Occurrence or absence of outliers on the FHR trace due to accelerations and decelerations controlled by vagal function

Formation of accelerations and decelerations controlled by vagal function
Reddy et al., 2009

Signorini et al., 2005

Signorini et al., 2005

Fanelli et al., 2013; Signorini et al., 2014

Fanelli et al., 2013; Signorini

Van Leeuwen et al., 1999; Lange et al., 2005

Van Leeuwen et al., 1999; Lange et al., 2005

Van Leeuwen et al., 1999; Lange et al., 2005

Lange et al., 2005; Tetschke et al., 2016

Tetschke et al., 2016; Marzbanrad et al., 2017

Tetschke et al., 2016;

Marzbanrad et al., 2017

Tetschke et al., 2016; Hoyer et al., 2017

Van Leeuwen et al., 1999 et al., 2014
Regularity and complexity on the interbeat sequence. Complexity increases as gestation progress due to maturation of the parasympathetic system
Calculated with the cardiovascular toolbox (Vest et al., 2018), setting the $m$ and $r$ parameters in 2 and 0.1 of the standard deviation of the input signal 
TABLE 3 | Continued

\begin{tabular}{|c|c|c|c|}
\hline Feature & Calculation & Interpretation & Relevant previous works \\
\hline Fractal dimension & $\begin{array}{l}\text { Calculated using the Higuchi's algorithm (Higuchi, } \\
\text { 1988), setting the interval parameters as } 5\end{array}$ & $\begin{array}{l}\text { Occurrence of recursive patterns on the interbeat } \\
\text { sequence }\end{array}$ & Kikuchi et al., 2005 \\
\hline Lyapunov exponent & $\begin{array}{l}\text { Calculated following steps presented in Rosenstein } \\
\text { et al. (1993) setting embedding dimension and lag } \\
\text { parameters as } 1 \text { and } 2\end{array}$ & $\begin{array}{l}\text { Dynamics properties (contraction or expansion) of } \\
\text { the interbeat sequence due to vagal function }\end{array}$ & Van Leeuwen et al., 1999 \\
\hline Tone & $\begin{array}{l}\text { Average of the percentile difference of successive beat } \\
\text { intervals }\end{array}$ & Measurement of the sympatho-vagal balance & Khandoker et al., 2015 \\
\hline Entropy & $\begin{array}{l}\text { Calculated by using the Shannon formula (Shannon, } \\
\text { 1948) on the percentile difference of successive beat } \\
\text { intervals distribution }\end{array}$ & Complexity of sympatho-vagal balance & Khandoker et al., 2015 \\
\hline $\begin{array}{l}\text { Generalized mutual } \\
\text { information (GMI) }\end{array}$ & $\begin{array}{l}\text { Calculated following steps in Hoyer et al. (2012), setting } \\
\text { dimension parameter at } 3 \text { and delay parameter at } 1\end{array}$ & Complexity of sympatho-vagal balance. & Hoyer et al., 2012, 2017 \\
\hline \multicolumn{4}{|c|}{ FREQUENCY-DOMAIN FEATURES } \\
\hline Low frequency (LF) & $\begin{array}{l}\text { Power spectral density of the FHR time series on the } \\
\text { band } 0.03-0.15 \mathrm{~Hz}\end{array}$ & $\begin{array}{l}\text { Associated with the sympathetic control and } \\
\text { vasomotor activity }\end{array}$ & $\begin{array}{l}\text { Signorini et al., 2003, 2014; } \\
\text { Van Leeuwen et al., } 2003\end{array}$ \\
\hline $\begin{array}{l}\text { Medium frequency } \\
\text { (MF) }\end{array}$ & $\begin{array}{l}\text { Power spectral density of the FHR time series on the } \\
\text { band } 0.15-0.50 \mathrm{~Hz}\end{array}$ & $\begin{array}{l}\text { Measurement of the fetal activity and mechanical } \\
\text { movement induced by maternal breathing }\end{array}$ & $\begin{array}{l}\text { Signorini et al., 2003, 2014; } \\
\text { Van Leeuwen et al., } 2003\end{array}$ \\
\hline High frequency (HF) & $\begin{array}{l}\text { Power spectral density of the FHR time series on the } \\
\text { band } 0.5-1 \mathrm{~Hz}\end{array}$ & $\begin{array}{l}\text { Associated with respiration controlled by vagal } \\
\text { activity }\end{array}$ & $\begin{array}{l}\text { Signorini et al., 2003, 2014; } \\
\text { Van Leeuwen et al., } 2003\end{array}$ \\
\hline$L F /(M F+H F)$ & Ratio between LF and the summation of MF and HF & $\begin{array}{l}\text { Fluctuation between physiological control } \\
\text { components and fetus activity level }\end{array}$ & $\begin{array}{l}\text { Signorini et al., 2003, 2014; } \\
\text { Van Leeuwen et al., } 2003\end{array}$ \\
\hline
\end{tabular}

The third column provides a physiological interpretation of the feature, and the fourth column displays relevant previous works in which the metrics have used for fetal maturity assessment.

TABLE 4 | Detail of maternal hemodynamic formulae calculated using the SBP, DBP and MHR taken with the self-inflating blood pressure device.

\begin{tabular}{lll}
\hline Metric name & Formula & References \\
\hline Pulse pressure (PP) & $S B P-D B P$ & Stouffer, 2008 \\
Mean arterial pressure (MAP) & $(S B P+D B P \times 2) / 3$ & Stouffer, 2008 \\
Cardiac output (CO) & $M H R \times P P \times 0.002$ & Hill et al., 2011 \\
Rate pressure product (RPP) & $M H R \times S B P$ & Robinson, 1967 \\
Shock index (SI) & $M H R / S B P$ & Singh et al., 2014 \\
Modified Shock Index (MSI) & $M H R / M A P$ & Singh et al., 2014 \\
Stroke volume (SV) & $C O / M H R$ & Stouffer, 2008 \\
\hline
\end{tabular}

TABLE 5 | Number of visits per gestational age (GA) for the 104 normal birth weight training set, the 25 normal birth weight test set, and the 24 low birth weight test set.

\begin{tabular}{lccc}
\hline $\begin{array}{l}\text { Gestational } \\
\text { age (months) }\end{array}$ & $\begin{array}{c}\text { Training set } \\
\text { Normal birth } \\
\text { weight }\end{array}$ & $\begin{array}{c}\text { Test set } \\
\text { Normal birth } \\
\text { weight }\end{array}$ & $\begin{array}{c}\text { Test set } \\
\text { Low birth } \\
\text { weight }\end{array}$ \\
\hline 6 & 6 & 3 & 3 \\
7 & 28 & 10 & 7 \\
8 & 32 & 5 & 5 \\
9 & 48 & 10 & 10 \\
\hline Total & 114 & 28 & 25 \\
\hline
\end{tabular}

between 0 and 1 , to one of the minority data points. In this study, the ADASYN was implemented as described in He et al. (2008).
Before training a model, the balanced training set and the held-out fold set were standardized by subtracting the mean of the respective feature vector and dividing it by its standard deviation computed in the training data only. This standardization method was selected as it has shown to be suitable for feature scaling in machine learning methods (Tax and Duin, 2000).

The 50-trial 5-fold cross-validation was assessed using three different regression approaches: Elastic Net, Support Vector Regression (SVR), and Gradient Boosting Tree (GBT). At each iteration of the cross-validation, the training folds were used to select the most relevant features for the SVR and GBT models. For the SVR, features were selected using the maximum relevance and minimum redundancy (mRMR) algorithm (Peng et al., 2005), which ranks the most relevant features based on mutual information gain. For the GBT model, the features were selected by training a GBT with 100 trees and learning rate of 1 on the training folds, and then identifying the most relevant features by summing the feature weights over all the weak learners.

To optimize each model's hyperparameters, a nested crossvalidation using a grid search on the training folds was used for each model. The grid search for the three models was defined as:

- Elastic Net. The linear penalty term, $\lambda$ was defined as $\{0.1,0.2, \ldots, 0.8,0.9\}$. The quadratic penalty term was given by $\frac{1-\lambda}{2}$. For each $\lambda$ value, a set of 100 values of regularization parameters were tested. The regularization parameter set was generated by first finding the largest value, $\theta$, that gave a non-null model (i.e., intercept $\neq 0$ ), and then the remaining 
99 values were defined by decreasing $\theta$ by $10^{-5}$, so that the ratio of the smallest to the largest value of the set was $10^{-4}$.

- SVR. The grid search for the soft margin $(C)$ and the margin of tolerance $(\epsilon)$ were defined as: $C \in\left\{2^{-3}, 2^{-1}, \ldots, 2^{8}\right\}$ and $\epsilon \in\left\{2^{-10}, 2^{-9}, \ldots, 2^{-5}\right\}$. The Gaussian radial basis function parameter $(\gamma)$ was analytically estimated as reported in Caputo et al. (2002). Namely, $\gamma$ was derived by calculating the distribution of $\left\|x-x^{\prime}\right\|^{2}$ between a subset containing $70 \%$ of the training set, and then taking the inverse of the median of this distribution.
- GBT. The grid search for the learning rate was defined as $\{0.1,0.25,0.5,1\}$ and the number of trees was defined as $\{100,150,200, \ldots, 500,550\}$. The number of maximum splits (tree height) was defined as $\left\{1,2, \ldots, \log _{2}(S-1)\right\}$; where $S$ is the total number of visits of the training folds.

\subsubsection{Analyzing the Training/Validation Output}

The 50 trial 5-fold cross-validation resulted in 50 median absolute error (MAE) vectors, and 250 selected feature vectors. From the 50 MAE vectors, the median, interquantile range, and the lower and upper 95\% confidence interval for the median were

TABLE 6 | Pearson correlation between the features and the gestational age in months.

\begin{tabular}{|c|c|c|c|c|c|c|}
\hline \multirow[t]{2}{*}{ Feature } & \multicolumn{2}{|c|}{ Overall } & \multicolumn{2}{|c|}{ Normal birth weight } & \multicolumn{2}{|c|}{ Low birth weight } \\
\hline & Correlation & $p$-value & Correlation & $p$-value & Correlation & $p$-value \\
\hline STV & -0.03 & 0.67 & -0.08 & 0.35 & 0.16 & 0.45 \\
\hline LTV & 0.06 & 0.45 & 0.04 & 0.67 & 0.14 & 0.52 \\
\hline AAC & 0.11 & 0.17 & 0.13 & 0.11 & -0.06 & 0.78 \\
\hline $\mathrm{BHR}^{\S}$ & -0.03 & 0.66 & 0.03 & 0.70 & -0.42 & 0.04 \\
\hline $\mathrm{DBP}^{\star \ddagger}$ & 0.32 & $<0.01$ & 0.32 & $<0.01$ & 0.35 & 0.09 \\
\hline $\mathrm{SBP}^{\star \star}$ & 0.30 & $<0.01$ & 0.32 & $<0.01$ & 0.16 & 0.44 \\
\hline $\mathrm{MHR}^{\ddagger}$ & 0.09 & 0.24 & 0.16 & 0.05 & -0.29 & 0.16 \\
\hline DAC & -0.09 & 0.24 & -0.14 & 0.11 & 0.17 & 0.41 \\
\hline \#Accelarations/minute ${ }^{\star \ddagger}$ & 0.19 & 0.01 & 0.19 & 0.02 & 0.20 & 0.34 \\
\hline ॥ & 0.05 & 0.53 & 0.07 & 0.44 & -0.06 & 0.79 \\
\hline$L T I^{\star \ddagger}$ & -0.24 & $<0.01$ & -0.26 & $<0.01$ & -0.07 & 0.73 \\
\hline ApEn & -0.13 & 0.09 & -0.12 & 0.17 & -0.24 & 0.26 \\
\hline LF & -0.08 & 0.33 & -0.10 & 0.23 & 0.08 & 0.71 \\
\hline $\mathrm{MF}^{\ddagger}$ & 0.14 & 0.07 & 0.20 & 0.02 & -0.22 & 0.29 \\
\hline HF & 0.06 & 0.46 & 0.08 & 0.33 & -0.09 & 0.68 \\
\hline LF/(MF + HF) & -0.08 & 0.31 & -0.11 & 0.19 & 0.10 & 0.64 \\
\hline varlS & 0.12 & 0.13 & 0.11 & 0.19 & 0.20 & 0.33 \\
\hline Fractal Dimension & -0.04 & 0.61 & -0.11 & 0.19 & 0.28 & 0.18 \\
\hline $\mathrm{mIS} \S$ & 0.02 & 0.79 & -0.04 & 0.61 & 0.40 & 0.05 \\
\hline stdIS ${ }^{\star \ddagger}$ & 0.18 & 0.02 & 0.18 & 0.03 & 0.20 & 0.34 \\
\hline rmssdls & 0.02 & 0.84 & -0.02 & 0.80 & 0.21 & 0.32 \\
\hline Lyapuno exponent ${ }^{\ddagger}$ & 0.14 & 0.07 & 0.17 & 0.04 & -0.08 & 0.71 \\
\hline skewnessls & -0.10 & 0.20 & -0.09 & 0.28 & -0.35 & 0.09 \\
\hline kurtosisls & -0.07 & 0.35 & -0.10 & 0.26 & 0.11 & 0.61 \\
\hline GMI & 0.09 & 0.26 & 0.13 & 0.14 & -0.12 & 0.58 \\
\hline Tone ${ }^{\ddagger}$ & -0.12 & 0.14 & -0.16 & 0.05 & 0.19 & 0.37 \\
\hline Entropy & 0.06 & 0.42 & 0.04 & 0.63 & 0.15 & 0.48 \\
\hline stdlS/rmssdls* & 0.20 & 0.01 & 0.24 & $<0.01$ & 0.01 & 0.96 \\
\hline PNN5 & 0.07 & 0.37 & 0.03 & 0.69 & 0.24 & 0.25 \\
\hline STV/LTV** & -0.21 & 0.01 & -0.24 & $<0.01$ & -0.04 & 0.85 \\
\hline PP & 0.06 & 0.48 & 0.08 & 0.32 & -0.11 & 0.60 \\
\hline $\mathrm{MAP}^{\star \ddagger}$ & 0.35 & $<0.01$ & 0.35 & $<0.01$ & 0.31 & 0.13 \\
\hline $\mathrm{CO}^{\ddagger}$ & 0.12 & 0.11 & 0.19 & 0.02 & -0.36 & 0.08 \\
\hline$R P P^{\star \star \ddagger}$ & 0.22 & $<0.01$ & 0.30 & $<0.01$ & -0.25 & 0.22 \\
\hline $\mathrm{SI}$ & -0.04 & 0.61 & 0.01 & 0.90 & -0.30 & 0.14 \\
\hline MSI & -0.06 & 0.47 & 0.00 & 0.98 & -0.35 & 0.09 \\
\hline SV & 0.06 & 0.48 & 0.08 & 0.32 & -0.11 & 0.60 \\
\hline
\end{tabular}

$A^{*}, \neq$, and $\$$ indicate if the correlation coefficient is statistically significant ( $p$-value $<0.05$ ) for overall newborns, normal birth weight newborns, and low birth weight newborns, respectively. 
determined. The median MAEs were compared to select which regression model (Elastict Net, SVR, or GBT) to use in the test stage.

From the 250 selected feature vectors, the top twenty most relevant features were identified. To that end, the features were ranked at each feature vectors, and the mean rank was determined by averaging the ranking of each feature over the 250 feature vectors. This simple aggregation technique was used as it has shown to be effective to combine different features sets in the medical application field (Saeys et al., 2007; Wu et al., 2018).

Using the top ten, top fifteen, and top twenty, the same validation/training procedure was repeated to identify the best performing feature set for estimating GA.

\subsubsection{Testing Methodology}

The model selected with the best performing feature set was then used to train a final model on the training set of normal birth weight patients. Before training the final model, the training data were balanced using ADASYN (He et al., 2008), and the parameters were optimized using grid search as explained in subsection 3.6.1. The final model was then used to estimate the GA for both the 25 held-out normal birth weight and the 24 LBW patients.

Since the final model depends on the nature of the synthetic data added to the training dataset, the testing procedure was performed 100 times to evaluate the variability of the model's performance. The median, interquantile range, and the lower and upper $95 \%$ confidence interval for the median were determined for the two test groups. To determine if there was any difference in GA estimation distribution of errors between the normal and LBW newborns, a two-sided Wilcox rank-sum test hypotheses test was evaluated on the data.

\subsection{Detecting Possible Cases of IUGR}

Since we are assuming that IUGR cases are those with LBW, the estimated GA for the test set were compared against birth weight. To that end, the GA error estimation was defined as the difference between the GA based on the LMP and the median GA estimation over the 100 repetition. Then, a robust least square was fitted using the birth weight as independent variable and the GA error estimation as the response variable.

Finally, the area under the receiver operating characteristic (AUROC) was calculated to discriminate between LBW and NBW newborns. The AUROC was calculated using the difference between LMP-based GA and the estimated GA. As we hypothesized that LBW newborns are likely to result in underestimations (LMP-based GA > estimated GA), a newborn was considered LBW if the difference between the LMP and SVR estimates of the GA was greater than the thresholds used in the AUROC analysis.

\section{RESULTS}

\subsection{Correlation Between Extracted Feature and Gestational Age}

Table 6 shows the Pearson correlation between the features and the gestational age. Nine out of the 37 extracted features showed
TABLE 7 | Mean absolute errors (MAE) of the 50 trial 5-fold cross validation for the Elastic Net, SVR, and Gradient boosting tree.

\begin{tabular}{llccccc}
\hline \multirow{2}{*}{ Model } & Metric & \multicolumn{5}{c}{ Gestational age (months) } \\
\cline { 3 - 7 } & & $\mathbf{6}$ & $\mathbf{7}$ & $\mathbf{8}$ & $\mathbf{9}$ & All \\
\hline Elastic Net & Median & 1.54 & 0.51 & 0.50 & 1.40 & 0.93 \\
& IQR & 0.10 & 0.04 & 0.07 & 0.14 & 0.08 \\
& LCl & 1.52 & 0.50 & 0.49 & 1.36 & 0.91 \\
& UCl & 1.58 & 0.52 & 0.52 & 1.42 & 0.96 \\
\hline \multirow{2}{*}{ SVR } & Median & 1.57 & 0.51 & 0.43 & 1.15 & 0.80 \\
& IQR & 0.17 & 0.09 & 0.10 & 0.11 & 0.06 \\
& LCl & 1.54 & 0.48 & 0.40 & 1.11 & 0.79 \\
& UCl & 1.65 & 0.54 & 0.47 & 1.17 & 0.83 \\
\hline \multirow{2}{*}{ Gradient boosting tree } & Median & 1.77 & 0.85 & 0.65 & 0.81 & 0.86 \\
& IQR & 0.42 & 0.20 & 0.14 & 0.18 & 0.08 \\
& LCl & 1.66 & 0.82 & 0.61 & 0.77 & 0.83 \\
& UCl & 1.93 & 0.89 & 0.70 & 0.89 & 0.88 \\
\hline
\end{tabular}

For each model, the median, interquantile range, and the $95 \%$ confidence interval for the median of the MAE are provided.

a statistically significant correlation with gestational age. For the normal birth weight, $38 \%$ of the features showed evidence of a significant correlation with gestational age, whereas, for the low birth newborns only two features were correlated. The correlation of fHRV indexes indicated a variation of such metrics during pregnancy, thus being relevant to assess fetal maturation for this project.

\subsection{Training/Validation Performance}

Table 7 shows the mean absolute error (MAE) for the 50 trial 5 -fold cross-validation. For all the three regression models, the MAE of the seventh and eighth gestational months were lower than those of the extreme months evaluated. The regression model with the lowest the overall median MAE over the 50 trials was the SVR with a value of 0.8 months. Furthermore, the SVR and Elastic Net were the models with the lowest interquartile range for the MAE over the 50 trials, thus indicating a lower variance of these to models in comparison to the GBT.

\subsection{Ranking the Features}

Table 8 shows the top twenty features for estimating GA based on the average ranking of the 250 feature vectors for the SVR. Seven out of the ten top features were derived from the 1D-Doppler ultrasound, from which fHRV linear indexes were among the most selected. Maternal blood pressure based features were also included in the top features, with MAP as the most selected feature of that group.

Table 9 presents the results obtained by repeating the training/validation procedure using the top ten, top fifteen, and top twenty features. The top fifteen feature set achieved the lowest median MAE over the 50 trials with a value of 0.76 months $(95 \%$ $\mathrm{CI}=0.75-0.78$ months). 
TABLE 8 | Feature ranking obtained after averaging the individual 250 feature ranking resulted in the 50 trial 5 -fold cross-validation.

\begin{tabular}{|c|c|c|}
\hline Ranking & Feature & Type \\
\hline \multirow[t]{10}{*}{ Top 10} & Tone & Non-linear and Complexity \\
\hline & \#acc/min & Linear \\
\hline & MAP & Hemodynamic formula \\
\hline & ApEn & Non-linear and Complexity \\
\hline & KurtosisIS & Linear \\
\hline & $\mathrm{CO}$ & Hemodynamic formula \\
\hline & STV/LTV & Linear \\
\hline & SkewnessIS & Linear \\
\hline & $\mathrm{PP}$ & Hemodynamic formula \\
\hline & LTI & Linear \\
\hline \multirow[t]{5}{*}{ Top 15} & stdIS/rmssdlS & Linear \\
\hline & DBP & Hemodynamic formula \\
\hline & $\mathrm{RPP}$ & Hemodynamic formula \\
\hline & varlS & Linear \\
\hline & stdIS & Linear \\
\hline \multirow[t]{5}{*}{ Top 20} & GMI & Non-linear and Complexity \\
\hline & $\|$ & Linear \\
\hline & DAC & Linear \\
\hline & MF & Spectral \\
\hline & basal FHR & Linear \\
\hline
\end{tabular}

TABLE 9 | Mean absolute errors (MAE) of the 50 trial 5 -fold cross validation for the SVR using the top10, top15, and top20 features.

\begin{tabular}{llccccc}
\hline Model & Metric & \multicolumn{5}{c}{ Gestational age (months) } \\
\cline { 3 - 7 } & & $\mathbf{6}$ & $\mathbf{7}$ & $\mathbf{8}$ & $\mathbf{9}$ & All \\
\hline SVR & Median & 1.62 & 0.48 & 0.43 & 1.18 & 0.82 \\
(top 10 features) & IQR & 0.18 & 0.10 & 0.09 & 0.12 & 0.07 \\
& LCl & 1.57 & 0.45 & 0.40 & 1.13 & 0.80 \\
& UCl & 1.67 & 0.50 & 0.45 & 1.21 & 0.83 \\
\hline SVR & Median & 1.51 & 0.47 & 0.44 & 1.04 & 0.76 \\
(top 15 features) & IQR & 0.17 & 0.08 & 0.07 & 0.15 & 0.07 \\
& LCl & 1.44 & 0.45 & 0.42 & 1.01 & 0.75 \\
& UCl & 1.54 & 0.50 & 0.46 & 1.08 & 0.78 \\
\hline SVR & Median & 1.56 & 0.45 & 0.43 & 1.16 & 0.81 \\
(top 20 features) & IQR & 0.15 & 0.10 & 0.08 & 0.09 & 0.05 \\
& LCl & 1.52 & 0.42 & 0.40 & 1.14 & 0.79 \\
& UCl & 1.60 & 0.47 & 0.44 & 1.20 & 0.81 \\
\hline
\end{tabular}

For each model, the median, interquantile range, and the 95\% confidence interval for the median of the MAE are provided.

\subsection{Testing Performance}

Table 10 shows the performance of the 100 repetitions of the SVR with the top fifteen tested on the held-out 25 normal birth weight newborns and the $24 \mathrm{LBW}$ newborns. The median MAE for each gestational month and the overall was statistically significantly
TABLE 10 | Mean absolute errors (MAE) of the 100 trials on the test (held-out) normal birth weight and LBW newborns.

\begin{tabular}{lllllll}
\hline \multirow{2}{*}{ Newborn type } & Metric & \multicolumn{5}{c}{ Gestational age (months) } \\
\cline { 3 - 7 } & & $\mathbf{6}^{\dagger}$ & $\mathbf{7}^{\dagger}$ & $\mathbf{8}^{\dagger}$ & $\mathbf{9}^{\dagger}$ & All $^{\dagger}$ \\
\hline Normal birth weight & Median & 1.06 & 0.53 & 0.33 & 0.99 & 0.72 \\
& IQR & 0.25 & 0.05 & 0.07 & 0.07 & 0.05 \\
& LCl & 1.03 & 0.52 & 0.32 & 0.98 & 0.71 \\
& UCl & 1.08 & 0.54 & 0.35 & 1.00 & 0.72 \\
\hline \multirow{2}{*}{ Low birth weight } & Median & 1.26 & 0.73 & 0.68 & 1.32 & 1.01 \\
& IQR & 0.11 & 0.05 & 0.09 & 0.06 & 0.03 \\
& LCI & 1.24 & 0.72 & 0.67 & 1.30 & 1.01 \\
& UCl & 1.29 & 0.74 & 0.69 & 1.33 & 1.02
\end{tabular}

For each type of newborns, the median, interquantile range, and the 95\% confidence interval for the median of the MAE are provided. $A^{+}$indicates a significant difference between the median GA estimations of the normal and LBW newborns for the 100 repetitions (two-sided Wilcox rank-sum test; $\alpha=0.05$ ).

higher for the LBW newborns (two-sided Wilcox rank-sum test; $\alpha=0.05)$. The difference between the median MAE for the two groups was increasing throughout the GA, resulting in a difference of 0.29 month for the overall estimation.

Figure 3 shows the difference $(\delta)$ between GA based on the LMP and the median estimated GA over the 100 repetitions for each visit. The LBW newborns' (red crosses) GAs were generally overestimated (LMP-GA < estimated GA) for the eighth and ninth gestational months compared to the normal birth weight newborns (blue circles). For the eighth and ninth gestational months, on the other hand, the LBW newborns were generally underestimated (LMP-GA > estimated GA) compared to the normal birth weight newborns.

For newborns with more than one visit, Figure 3 shows a line connecting the median error across GA. For both normal and LBW newborns, the GA estimates trended from overestimation to underestimations as GA increased. However, the discrepancy was higher for the LBW newborn with a difference of around 2.5 months between the seventh and the ninth gestational month. In contrast, the maximum difference for normal birth weight newborns was $\sim 0.75$ month.

Figure 4 shows the median of the estimated GA for each label of the LMP method. For all the gestational months, the LBW group resulted in a lower median GA estimations than the normal birth weight group. The median difference between normal and LBW newborns is greater for the last 2 months of pregnancy, thus indicating a higher inconsistency in the features for GA estimation between the type of newborns from the eighth month of gestation onward.

\subsection{GA Estimation Errors as a Function of Birth Weight}

Figure 5 shows the GA estimation errors over estimated birth weight for the tested newborns. Robust least-square fits were performed for each type of newborn, as well as for all the newborns as a whole. All the fits provided negative slopes and 


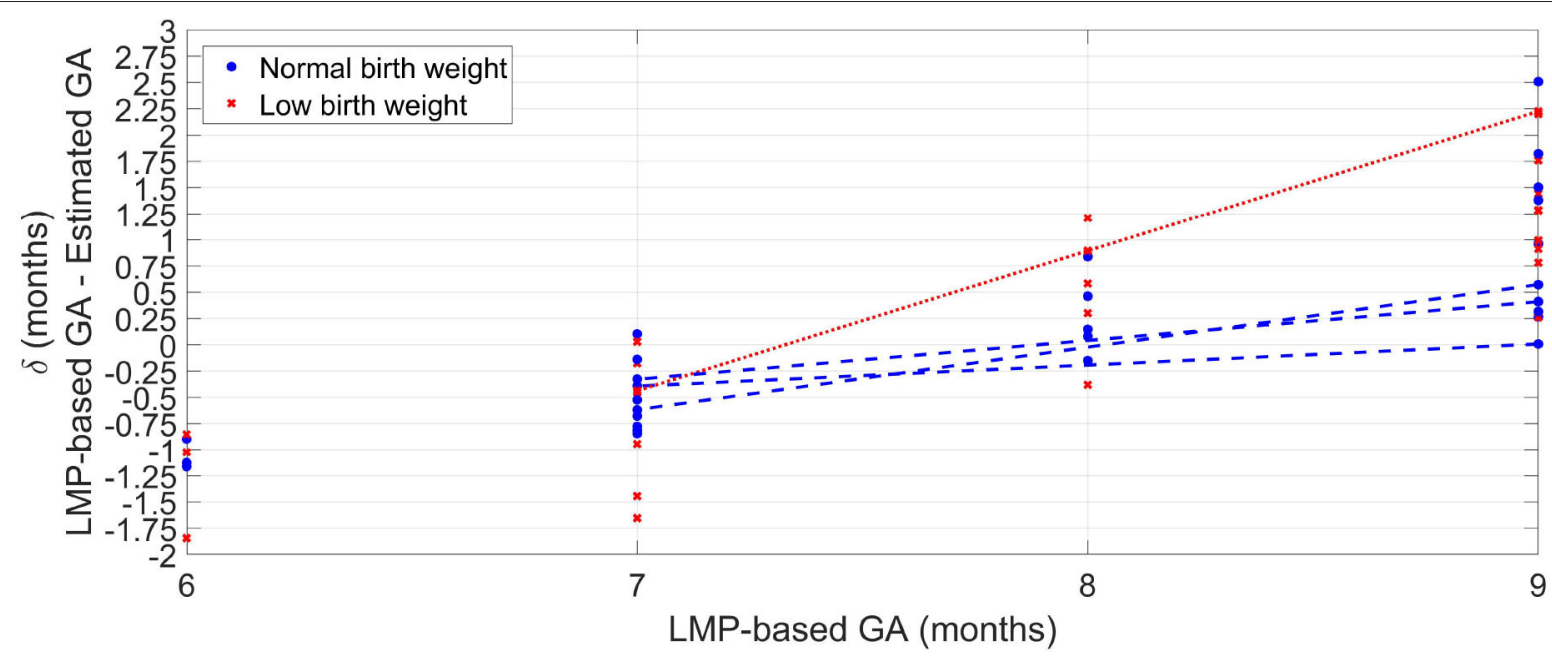

FIGURE 3 | Median error of the 100 repetitions against GA provided by the LMP method for the normal weight newborns (blue circles) and the LBW newborns (red crosses). For newborns with more than one visit, a line connects the median error along with the GA.

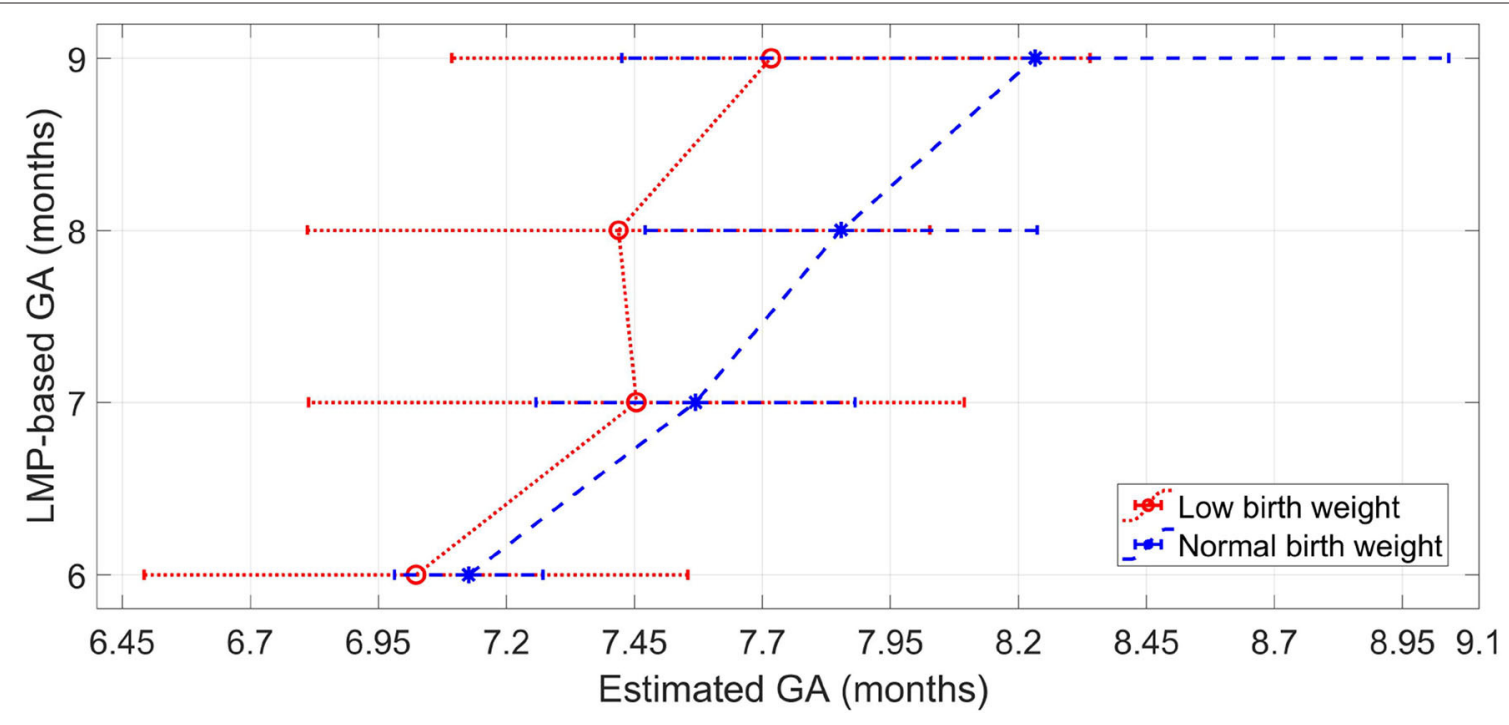

FIGURE 4 | Median and interquartile range of the estimated GA for each label of the LMP method for the normal weight newborns and the LBW newborns.

negative Pearson correlation values $(\rho)$. The inverse relationship between GA estimation error and birth weight indicates that there are more underestimations for newborns with LBW. In fact, for the LBW newborns, fifteen visits achieved underestimations, whereas ten visits obtained overestimations. For the normal weight newborns, the fitted line was $\delta_{N B W}=1.85-$ $0.60 w_{N B W}(\rho=-0.15, P$-value $=0.45)$. For the low weight newborns, the fitted line was $\delta_{L B W}=0.82-0.23 w_{L B W}(\rho=$ $-0.06, P$-value $=0.76)$. For all the newborns, the fitted line was $\delta=1.12-0.35 w(\rho=-0.13, P$-value $=0.37)$.

The AUROC using the estimation difference between NBW and LBW resulted in 0.55. However, as in Figure 5 is observable than there was a more remarkable difference between newborns with weight lower than $2.3 \mathrm{~kg}$, and those with a weight $>3.1$ $\mathrm{kg}$, the ROC analysis was repeated for these two groups. This comparison yielded in an AUROC of 0.63 , thus suggesting acceptable discrimination for these two groups.

\section{DISCUSSION}

\subsection{Interpretations of Findings}

The results presented in this work indicate that it is possible to provide a proxy for screening fetal growth retardation in a resource-constrained setting by using the difference between GA estimated by LMP and the GA estimated from features extracted from an inexpensive Doppler transducer and a blood pressure device. 


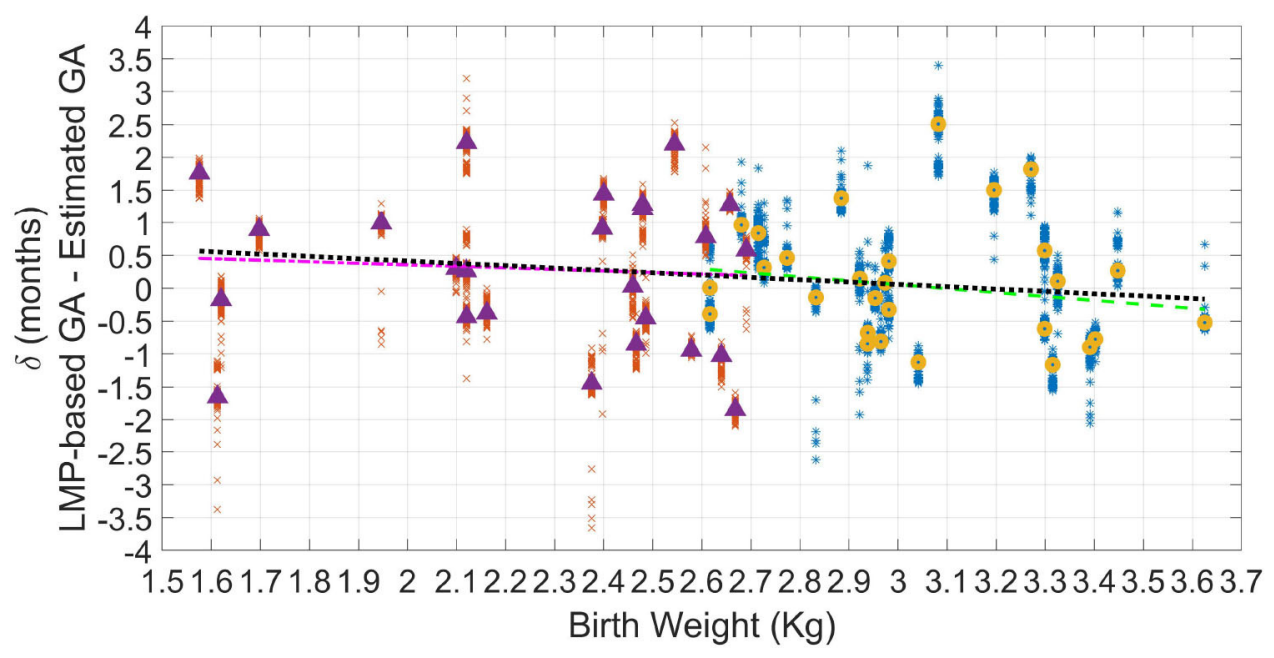

* NBW errors LBW errors

- median NBW errors

$\Delta$ median LBW errors

- - NBW RLS

----LBW RLS

.....All RLS

FIGURE 5 | Error in GA from 100 repetitions $(\delta)$ as the difference between GA based on the LMP and the estimated GA. The median error of the 100 repetitions for each recording (or visit) is displayed (triangles for LBW newborns; and circles for normal birth weight newborns). Robust least square fits are also shown.

This proxy fetal assessment relies on the GA estimation approach introduced in this work, which using a pregnancy conversion factor of $\frac{40 \text { weeks }}{9 \text { months }}$ resulted in a median MAE of 3.2 and 4.5 weeks for the normal and LBW newborns, respectively. Interestingly, these MAE values are comparable to those presented in Marzbanrad et al. (2016) and Marzbanrad et al. (2017) of 2.7-5.1 weeks obtained using a step-wise regression using $1 \mathrm{D}$-DUS and fECG signals recorded by medical professionals in a high-resource/high-income country. Moreover, unlike Tetschke et al. (2016), our work did not require high-resolution input signals, making the implementation of the approach described here feasible in LMICs. Notably, our GA estimations were lower than the 6 weeks error associated with symphysis-fundal height, which is the common dating method used in LMICs to detect IUGR (Griffiths et al., 2008).

The higher GA estimation errors for the LBW newborns indicate that this type of patient has different patterns in the $1 \mathrm{D}$ DUS and maternal blood pressure features than normal birth weight newborns of the same GA. We note that this difference is related to the birth weight as the AUROC between very low birth weight $(<2.3 \mathrm{~kg})$ and normal birth weight indicated acceptable discrimination. Therefore, assuming that LBW is a consequence of IUGR (Lee et al., 2013), a potential sign of IUGR can be detected when the estimated GA is lower than the GA calculated from the LMP. This provides evidence to indicate that our method is an alternative and low-cost fetal growth assessment approach for identifying cases that need to be referred for further medical assessment in LMICs, in which symphysis-fundal height measurement is not sufficiently accurate (World Health Organization, 2016), and ultrasound imaging is not available (World Health Organization, 2014).

The longitudinal changes in the difference between GA estimations of low and normal birth weight newborns across gestation suggests that IUGR is progressive and is more evident for the eighth and ninth gestational months, as is shown in Figure 4 for patient with more than one visit. Therefore, our proxy method may be more effective in detecting fetal growth abnormalities during the last 2 months of gestation, thus helping to identify fetuses that need assistance during delivery to reduce adverse perinatal outcomes.

Another interesting finding was the selected features for estimating GA. The top fifteen features (Table 8) were consistent with features previous work for assessing gestational development (Van Leeuwen et al., 1999, 2003; Signorini et al., 2003; Wakai, 2004; Lange et al., 2005; Khandoker et al., 2009). Specifically, linear, non-linear and complexity features, such as tone, the number of accelerations per minute, approximate entropy, and statistical moments of the interbeat sequence, were the features which provided the SVR with the highest performance boost. The feature selection algorithm also demonstrated the potential of blood-pressure-derived features. This selection was relevant as little research has used this type of features for assessing fetal maturity. Finally, the STV/LTV ratio, which previously has shown to be relevant for detecting IUGR cases (Stroux et al., 2017), was also relevant for GA estimation.

\subsection{Study Limitations}

It should be noted that in this work possible cases of IUGR were defined by newborns birth weight. This assumption could not be validated as patients did not receive an ultrasound imaging exam to detect IUGR based on obstetrician standards. Moreover, LBW also includes small for gestational age and appropriate for gestational age/premature newborns ( $<37$ weeks). Presumably, premature and IUGR newborns behave similarly, and there is overlap between these two groups (Lee et al., 2013). Thus, our proxy method may be affected because LBW newborns do not fully capture all the IUGR population since such definition misses infants born small for gestational age above the 2,500 g cutoff and those who are both preterm and small for gestational age. Nevertheless, using LBW as a surrogate for IUGR was based on the fact that in LMICs around 60\% of the LBW is caused by IUGR (Lee et al., 2013). 
The method presented here estimates GA using the LMP method as a reference. As LMP is not a completely unbiased method for dating fetuses (Andersen et al., 1981; Dietz et al., 2007), our results may contain a bias. Moreover, since the errors in GA estimation provided by our method were larger than 2 weeks [the estimation error recommended in the literature (MacGregor and Sabbagha, 2008)], our method is not accurate enough to be used as a primary method for dating GA. However, for rural areas in LMICS, in which there is a lack of ultrasound imaging equipment and obstetricians, our method is a proxy for screening fetuses with possible abnormalities (LBW or IUGR) that need to be referred for further medical diagnosis and treatment. Superiority to the symphysis-fundal height measurement indicates that this method should be preferred.

Another limitation of this work was that the GA was recorded by the clinical team in months rather than weeks (Martinez et al., 2017). However, as a month includes a variable number of days, this introduces a quantization/rounding error-fetuses just a few days apart that fall into different months will look similar but will be identified as different. This decreases the accuracy of any model fitted to the data, resulting in larger absolute errors for the sixth and ninth gestational months.

This error can be thought of as a higher intra-class variance. When intra-class variance is high, it is recommended to use a longitudinal approach rather than cross-sectional one (Diggle et al., 2002). However, those models need multiple points per subject, in order to be able to apply mixed models considering the random effects of each individual. In this study we could not apply a longitudinal approach because the majority of subjects contained only one valid visit. Nevertheless, the MAE values obtained for our approach suggests that features and methods used here are promising for estimating GA based on the LMP method, which is a low-cost, feasible method to date pregnancy in LMICs (Neufeld et al., 2006).

Our study also included visits that were between the sixth and ninth months of gestation. To fully assess the capacity of our approach to estimating GA, it should be evaluate on metrics recorded in the first and second trimesters. Such an evaluation would allow for the comparison of our GA estimation in a fetal development period in which genetic and biological variability of fetal size is low, and in which Doppler images methods estimate GA more accurately (Reece et al., 1989).

We note that the analysis in this study applies only to singleton pregnancies. Non-singleton pregnancies were not assessed in this study and growth rates observed in these fetuses may well be divergent, or asymmetric.

Finally, we note that the approach presented here did not consider fetal sex to estimate GA. Although it may influence fHRV metrics used here for GA estimation, we deliberately avoid gender because the aim is to avoid the use of imaging Doppler, and sex determination, which present significant cost and social problems, respectively.

\subsection{Future Directions}

Future research should focus on increasing the temporal resolution of the GA labels (by recording the week of the LMP through community surveys perhaps), and use a more accurate dating method, such as expert-driven ultrasound imaging.

Future research should also evaluate the efficacy of the proxy presented here on confirmed diagnoses of IUGR. This evaluation would allow a full end-to-end assessment of how 1D-DUS and maternal blood pressure can contribute to detection fetal growth abnormalities.

Moreover, future research should ensure the collection of multiple visits during the course of pregnancy (and extending this to earlier gestational periods), so that a longitudinal analysis can be performed that incorporates the individual dynamics into the model.

\section{CONCLUSION}

This work introduced a proxy to detect possible cases of IUGR for constrained-resource environments in which ultrasound imaging is not available, and current low-cost methods are prone to error. The potential IUGR cases are detected by comparing GA based on the last menstrual period with estimates obtained 1DDUS and maternal blood pressure recordings collected with inexpensive devices, usable with little training. The method is valuable to endow non-medically trained operators with an objective metric to identify cases that need to be referred to further medical assistance. The assessment system may, therefore, have an immediate impact if coupled with suitable intervention, such as nutritional supplementation. However, a prospective clinical trial is required to show the efficacy of such metrics and intervention.

\section{DATA AVAILABILITY STATEMENT}

The datasets generated for this study will not be made publicly available. The data was collected in Guatemala rural community, including recordings of a vulnerable indigenous population. We need to obtain permission from the ethical committee before making the dataset publicly available.

\section{ETHICS STATEMENT}

The studies involving human participants were reviewed and approved by the Institutional Review Boards of Emory University, the Wuqu' Kawoq|Maya Health Alliance, and Agnes Scott College (Ref: IRB00076231-Mobile Health Intervention to Improve Perinatal Continuum of Care in Guatemala) and registered on ClinicalTrials.gov (identifier NCT02348840). The patients/participants provided their written informed consent to participate in this study.

\section{AUTHOR CONTRIBUTIONS}

GC, RH-C, and PR conceived of the study and acquired the funding. $\mathrm{RH}-\mathrm{C}$ and $\mathrm{PR}$ coordinated the data collection process in the Guatemalan rural community. CV, FM, and GC contributed to the development of techniques and analysis tools. The data was analyzed by CV, FM, and GC. CV implemented the methods 
presented here for estimating gestational age. CV, FM, PR, and GC contributed to the writing of the manuscript. All authors reviewed and approved the final manuscript.

\section{FUNDING}

CV was funded by a Fulbright Scholarship. GC, PR, and $\mathrm{RH}-\mathrm{C}$ acknowledge the support of the National Institutes

\section{REFERENCES}

Alexander, G. R., Tompkins, M. E., Petersen, D. J., Hulsey, T. C., and Mor, J. (1995). Discordance between LMP-based and clinically estimated gestational age: implications for research, programs, and policy. Public Health Rep. 110:395.

Andersen, H. F., Johnson, T. R. Jr., Flora, J. D. Jr., and Barclay, M. L. (1981). Gestational age assessment: II. Prediction from combined clinical observations. Am. J. Obstet. Gynecol. 140, 770-774. doi: 10.1016/0002-9378(81)90738-9

Andersson, S. (2011). Acceleration and deceleration detection and baseline estimation (Master's thesis), Chalmerss University of Technology, Göteborg, Sweden.

Batuwita, R., and Paladey, V. (2013). "Class imbalance learning methods for support vector machines," in Imbalanced Learning, Chapter 5, 1st Edn., eds H. He and Y. Ma (Piscataway, NJ: IEEE Press Editorial Board), 83-96. doi: 10.1002/9781118646106.ch5

Berkey, C. S., and Reed, R. B. (1987). A model for describing normal and abnormal growth in early childhood. Hum. Biol. 59, 973-987.

Caputo, B., Sim, K., Furesjo, F., and Smola, A. (2002). "Appearance-based object recognition using SVMs: which kernel should I use?” in Proceedings of NIPS Workshop on Statistical Methods for Computational Experiments in Visual Processing and Computer V, Vol. 2002 (Whistler, BC).

Cicolini, G., Pizzi, C., Palma, E., Bucci, M., Schioppa, F., Mezzetti, A., et al. (2011). Differences in blood pressure by body position (supine, fowler's, and sitting) in hypertensive subjects. Am. J. Hypertension 24, 1073-1079. doi: 10.1038/ajh.2011.106

Dawes, G., Houghton, C., and Redman, C. (1982). Baseline in human fetal heart-rate records. BJOG Int. J. Obstet. Gynaecol. 89, 270-275. doi: 10.1111/j.1471-0528.1982.tb04695.x

Dawes, G., Visser, G., Goodman, J., and Redman, C. (1981). Numerical analysis of the human fetal heart rate: the quality of ultrasound records. Am. J. Obstet. Gynecol. 141, 43-52. doi: 10.1016/0002-9378(81)90673-6

de Onis, M., Blössner, M., and Villar, J. (1998). Levels and patterns of intrauterine growth retardation in developing countries. Eur. J. Clin. Nutr. 52, S5-S15.

Dietz, P. M., England, L. J., Callaghan, W. M., Pearl, M., Wier, M. L., and Kharrazi, M. (2007). A comparison of LMP-based and ultrasound-based estimates of gestational age using linked California livebirth and prenatal screening records. Paediatr. Perinat. Epidemiol. 21, 62-71. doi: 10.1111/j.1365-3016.2007. 00862. $\mathrm{x}$

Diggle, P., Diggle, P. J., Heagerty, P., Liang, K.-Y., Heagerty, P. J., and Zeger, S. (2002). Analysis of Longitudinal Data. Oxford: Oxford University Press.

Dobbe, J., Lunshof, S., Boer, K., Wolf, H., and Grimbergen, C. (2001). The technique and algorithms for computerized analysis of longterm fetal heart rate recordings. Prenat. Neonat. Med. 6, 280-289. doi: 10.1080/13598630108500309

Fanelli, A., Magenes, G., Campanile, M., and Signorini, M. G. (2013). Quantitative assessment of fetal well-being through ctg recordings: a new parameter based on phase-rectified signal average. IEEE J. Biomed. Health Inform. 17, 959-966. doi: 10.1109/JBHI.2013.2268423

Frasch, M., Müller, T., Wicher, C., Weiss, C., Löhle, M., Schwab, K., et al. (2007). Fetal body weight and the development of the control of the cardiovascular system in fetal sheep. J. Physiol. 579, 893-907. doi: 10.1113/jphysiol.2006.124800

Griffiths, A., Pinto, A., and Margarit, L. (2008). A survey of methods used to measure symphysis fundal height. J. Obstet. Gynaecol. 28, 692-694. doi: $10.1080 / 01443610802462092$ of Health, the Fogarty International Center, and the Eunice Kennedy Shriver National Institute of Child Health and Human Development, grant number 1R21HD084114-01 (Mobile Health Intervention to Improve Perinatal Continuum of Care in Guatemala). GC has financial interest in Alivecor Inc. and receives unrestricted funding from the company. GC was also the CTO of Mindchild Medical and has ownership interests in Mindchild Medical.
He, H., Bai, Y., Garcia, E. A., and Li, S. (2008). "ADASYN: adaptive synthetic sampling approach for imbalanced learning," in 2008 IEEE International Joint Conference on Neural Networks (IEEE World Congress on Computational Intelligence) (Hong Kong: IEEE), 1322-1328.

Higuchi, T. (1988). Approach to an irregular time series on the basis of the fractal theory. Phys. D Nonlin. Phenom. 31, 277-283. doi: 10.1016/0167-2789(88)90081-4

Hill, L., Sollers, I., and Thayer, J. (2011). Evaluation of a simple estimation method for the derivation of cardiac output from arterial blood pressure and heart rate. Biomed. Sci. Instr. 48, 165-170.

Hoyer, D., Żebrowski, J., Cysarz, D., Gonçalves, H., Pytlik, A., Amorim-Costa, C., et al. (2017). Monitoring fetal maturation-objectives, techniques and indices of autonomic function. Physiol. Meas. 38:R61. doi: 10.1088/1361-6579/aa5fca

Hoyer, D., Nowack, S., Bauer, S., Tetschke, F., Rudolph, A., Wallwitz, U., et al. (2012). Fetal development of complex autonomic control evaluated from multiscale heart rate patterns. Am. J. Physiol. Heart Circ. Physiol. 304, R383R392. doi: 10.1152/ajpregu.00120.2012

Hoyer, D., Schmidt, A., Gustafson, K. M., Lobmaier, S. M., Lakhno, I., van Leeuwen, P., et al. (2019). Heart rate variability categories of fluctuation amplitude and complexity: diagnostic markers of fetal development and its disturbances. Physiol. Meas. 40:064002. doi: 10.1088/1361-6579/ab205f

Huhn, E., Lobmaier, S., Fischer, T., Schneider, R., Bauer, A., Schneider, K., et al. (2011). New computerized fetal heart rate analysis for surveillance of intrauterine growth restriction. Prenat. Diagn. 31, 509-514. doi: $10.1002 / p d .2728$

Kac, G., Mendes, R. H., Farias, D. R., Eshriqui, I., Rebelo, F., Benaim, C., et al. (2015). Hepatic, renal and inflammatory biomarkers are positively associated with blood pressure changes in healthy pregnant women: a prospective cohort. Medicine 94:683. doi: 10.1097/MD.0000000000000683

Kapaya, H., Jacques, R., and Anumba, D. (2018). Comparison of diurnal variations, gestational age and gender related differences in fetal heart rate (FHR) parameters between appropriate-for-gestational-age (AGA) and smallfor-gestational-age (SGA) fetuses in the home environment. PLoS ONE 13:e0193908. doi: 10.1371/journal.pone.0193908

Karl, S., Suen, C. S. L. W., Unger, H. W., Ome-Kaius, M., Mola, G., White, L., et al. (2015). Preterm or not-an evaluation of estimates of gestational age in a cohort of women from rural Papua New Guinea. PLoS ONE 10:e0124286. doi: 10.1371/journal.pone.0124286

Khandoker, A., Karmakar, C., Kimura, Y., Endo, M., Oshio, S., and Palaniswami, M. (2015). Tone entropy analysis of foetal heart rate variability. Entropy 17, 1042-1053. doi: 10.3390/e17031042

Khandoker, A. H., Kimura, Y., Ito, T., Sato, N., Okamura, K., and Palaniswami, M. (2009). Antepartum non-invasive evaluation of opening and closing timings of the cardiac valves in fetal cardiac cycle. Med. Biol. Eng. Comput. 47, 1075-1082. doi: 10.1007/s11517-009-0528-y

Kikuchi, A., Unno, N., Horikoshi, T., Shimizu, T., Kozuma, S., and Taketani, Y. (2005). Changes in fractal features of fetal heart rate during pregnancy. Early Hum. Dev. 81, 655-661. doi: 10.1016/j.earlhumdev.2005.01.009

Kluttig, A., Tiller, D., Schumann, B., Kuss, O., Greiser, K., Werdan, K., et al. (2010). Blood pressure measurement: differences between arm side, sitting and supine position and between consecutive measurements. Das Gesundh. 72:V215. doi: 10.1055/s-0030-1266407

Lange, S., Van Leeuwen, P., Geue, D., Hatzmann, W., and Grönemeyer, D. (2005). Influence of gestational age, heart rate, gender and time of day on fetal heart rate variability. Med. Biol. Eng. Comput. 43, 481-486. doi: 10.1007/BF02344729 
Lee, A. C., Katz, J., Blencowe, H., Cousens, S., Kozuki, N., Vogel, J. P., et al. (2013). National and regional estimates of term and preterm babies born small for gestational age in 138 low-income and middle-income countries in 2010. Lancet Glob. Health 1, e26-e36. doi: 10.1016/S2214-109X(13)70006-8

Lunghi, F., Magenes, G., Pedrinazzi, L., and Signorini, M. G. (2005). Detection of fetal distress though a support vector machine based on fetal heart rate parameters. Comput. Cardiol. 2005, 247-250. doi: 10.1109/CIC.2005.1588083

MacGregor, S. N., and Sabbagha, R. (2008). Assessment of gestational age by ultrasound. Glob. Libr. Women's Med. (2008). doi: 10.3843/GLOWM.10206

Malhotra, N., Malhotra, J., Mathur, V., Tomar, S., Singh, K., Rao, J., et al. (2014). "Antenatal assessment of fetal well-being," in Ultrasound in Obstetrics and Gynecology, Chapter 26, eds N. Malhotra, P. Kumar, S. Panchal, K. Shah, P. Acharya, and J. Malhotra (London: JP Medical Ltd.), 227-241. doi: 10.5005/jp/books/12162_26

Mantel, R., Van Geijn, H., Caron, F., Swartjes, J., Van Woerden, E., and Jongsma, H. (1990). Computer analysis of antepartum fetal heart rate: 1. Baseline determination. Int. J. Biomed. Comput. 25, 261-272. doi: 10.1016/0020-7101(90)90030-X

Martinez, B., Coyote, E., Hall-Clifford, R., Juarez, M., Miller, A. C., Francis, A., et al. (2018). mHealth intervention to improve the continuum of maternal and perinatal care in rural Guatemala: a pragmatic, randomized controlled feasibility trial. Reprod. Health 15:120. doi: 10.1186/s12978-018-0554-Z

Martinez, B., Hall-Clifford, R., Coyote, E., Stroux, L., Valderrama, C. E., Aaron, C., et al. (2017). Agile development of a smartphone app for perinatal monitoring in a resource-constrained setting. J. Health Inform. Dev. Countr. 11.

Marzbanrad, F., Khandoker, A., Kimura, Y., Palaniswami, M., and Clifford, G. D. (2016). "Estimating fetal gestational age using cardiac valve intervals," in Computing in Cardiology Conference (CinC) (Vancouuver, BC: IEEE), 109-112. doi: 10.22489/CinC.2016.035-146

Marzbanrad, F., Khandoker, A. H., Kimura, Y., Palaniswami, M., and Clifford, G. D. (2017). Assessment of fetal development using cardiac valve intervals. Front. Physiol. 8:313. doi: 10.3389/fphys.2017.00313

MSPAS/Guatemala, INE/Guatemala, Segeplán/Guatemala, and ICF International (2017). Encuesta Nacional de Salud Materno Infantil 2014-2015: Informe Final. Retrieved from: https://dhsprogram.com/publications/publication-fr318-dhsfinal-reports.cfm (accessed October 11, 2019).

Netea, R., Lenders, J., Smits, P., and Thien, T. (2003). Both body and arm position significantly influence blood pressure measurement. J. Hum. Hypertension 17:459. doi: 10.1038/sj.jhh.1001573

Neufeld, L. M., Haas, J. D., Grajéda, R., and Martorell, R. (2006). Last menstrual period provides the best estimate of gestation length for women in rural Guatemala. Paediatr. Perinat. Epidemiol. 20, 290-298. doi: 10.1111/j.1365-3016.2006.00741.x

Nyboe, E. L. (2011). An algorithm based on the Dawes/Redman criteria for automated fetal heart rate analysis (Master's thesis), Chalmerss University of Technology, Göteborg, Sweden.

Pardey, J., Moulden, M., and Redman, C. W. (2002). A computer system for the numerical analysis of nonstress tests. Am. J. Obstet. Gynecol. 186, 1095-1103. doi: $10.1067 / \mathrm{mob} .2002 .122447$

Peng, H., Long, F., and Ding, C. (2005). Feature selection based on mutual information criteria of max-dependency, max-relevance, and minredundancy. IEEE Trans. Pattern Anal. Mach. Intell. 27, 1226-1238. doi: 10.1109/TPAMI.2005.159

Peter, J. R., Ho, J. J., Valliapan, J., and Sivasangari, S. (2015). Symphysial fundal height (SFH) measurement in pregnancy for detecting abnormal fetal growth. Cochrane Database Syst. Rev. 2015:CD008136. doi: 10.1002/14651858.CD008136.pub3

Rebelo, F., Farias, D. R., Mendes, R. H., Schlüssel, M. M., and Kac, G. (2015). Blood pressure variation throughout pregnancy according to early gestational BMI: a Brazilian cohort. Arq. Brasil. Cardiol. 104, 284-291. doi: 10.5935/abc.20150007

Reddy, A., Moulden, M., and Redman, C. W. (2009). Antepartum high-frequency fetal heart rate sinusoidal rhythm: computerized detection and fetal anemia. Am. J. Obstet. Gynecol. 200, 407-e1. doi: 10.1016/j.ajog.2008.10.026

Reece, E. A., Gabrielli, S., Degennaro, N., and Hobbins, J. C. (1989). Dating through pregnancy: a measure of growing up. Obstet. Gynecol. Survey 44, 544-555. doi: 10.1097/00006254-198907000-00010

Rijken, M. J., De Livera, A. M., Lee, S. J., Boel, M. E., Rungwilailaekhiri, S., Wiladphaingern, J., et al. (2014). Quantifying low birth weight, preterm birth and small-for-gestational-age effects of malaria in pregnancy: a population cohort study. PLoS ONE 9:e100247. doi: 10.1371/journal.pone.0100247

Robinson, B. F. (1967). Relation of heart rate and systolic blood pressure to the onset of pain in angina pectoris. Circulation 35, 1073-1083. doi: 10.1161/01.CIR.35.6.1073

Romagnoli, S., Sbrollini, A., Burattini, L., Marcantoni, I., Morettini, M., and Burattini, L. (2019). Digital cardiotocography: What is the optimal sampling frequency? Biomed. Signal Process. Control 51, 210-215. doi: 10.1016/j.bspc.2019.02.016

Rosenstein, M. T., Collins, J. J., and De Luca, C. J. (1993). A practical method for calculating largest lyapunov exponents from small data sets. Phys. D Nonlin. Phenom. 65, 117-134. doi: 10.1016/0167-2789(93)90009-P

Saeys, Y., Inza, I., and Larrañaga, P. (2007). A review of feature selection techniques in bioinformatics. Bioinformatics 23, 2507-2517. doi: 10.1093/bioinformatics/btm344

Salas, S. P., Marshall, G., Gutiérrez, B. L., and Rosso, P. (2006). Time course of maternal plasma volume and hormonal changes in women with preeclampsia or fetal growth restriction. Hypertension 47, 203-208. doi: 10.1161/01.HYP.0000200042.64517.19

Schneider, U., Schleussner, E., Fiedler, A., Jaekel, S., Liehr, M., Haueisen, J., et al. (2009). Fetal heart rate variability reveals differential dynamics in the intrauterine development of the sympathetic and parasympathetic branches of the autonomic nervous system. Physiol. Meas. 30:215. doi: 10.1088/0967-3334/30/2/008

Shannon, C. (1948). A mathematical theory of communication. Bell Syst. Tech. J. 27, 379-423; 623-656. doi: 10.1002/j.1538-7305.1948.tb01338.x

Signorini, M. G., Fanelli, A., and Magenes, G. (2014). Monitoring fetal heart rate during pregnancy: contributions from advanced signal processing and wearable technology. Comput. Math. Methods Med. 2014:707581. doi: 10.1155/2014/707581

Signorini, M. G., Ferrario, M., Pedrinazzi, L., and Magenes, G. (2005). Analysis of echographic and heart rate time and frequency domain parameters for the antepartun fetal surveillance. Comput. Cardiol. 2005, 679-682. doi: 10.1109/CIC.2005.1588193

Signorini, M. G., Magenes, G., Cerutti, S., and Arduini, D. (2003). Linear and nonlinear parameters for the analysis of fetal heart rate signal from cardiotocographic recordings. IEEE Trans. Biomed. Eng. 50, 365-374. doi: 10.1109/TBME.2003.808824

Signorini, M. G., Pini, N., Malovini, A., Bellazzi, R., and Magenes, G. (2020). Integrating machine learning techniques and physiology based heart rate features for antepartum fetal monitoring. Comput. Methods Prog. Biomed. 185:105015. doi: 10.1016/j.cmpb.2019.105015

Singh, A., Ali, S., Agarwal, A., and Srivastava, R. N. (2014). Correlation of shock index and modified shock index with the outcome of adult trauma patients: a prospective study of 9860 patients. North Am. J. Med. Sci. 6:450. doi: 10.4103/1947-2714.141632

Steer, P. J., Little, M. P., Kold-Jensen, T., Chapple, J., and Elliott, P. (2004). Maternal blood pressure in pregnancy, birth weight, and perinatal mortality in first births: prospective study. BMJ 329:1312. doi: 10.1136/bmj.38258.566 262.7C

Stouffer, G. A. (ed.). (2008). "Useful hemodynamic formulas," in Cardiovascular Hemodynamics for the Clinician, Chapter Appendix 1, 1st Edn. (Malden, MA: Blackwell Futura), 289-292.

Stroux, L., Martinez, B., Coyote, E., King, N., Hall-Clifford, R., Rohloff, P., et al. (2016). An mhealth monitoring system for traditional birth attendant-led antenatal risk assessment in rural guatemala. J. Med. Eng. Technol. 40, 356-371. doi: 10.1080/03091902.2016.1223196

Stroux, L., Redman, C. W., Georgieva, A., Payne, S. J., and Clifford, G. D. (2017). Doppler-based fetal heart rate analysis markers for the detection of early intrauterine growth restriction. Acta Obstet. Gynecol. Scand. 96, 1322-1329. doi: 10.1111/aogs.13228

Tax, D. M., and Duin, R. P. (2000). Feature Scaling in Support Vector Data Descriptions. Technical report, American Association for Artificial Intelligence, Delft.

Tetschke, F., Schneider, U., Schleussner, E., Witte, O. W., and Hoyer, D. (2016). Assessment of fetal maturation age by heart rate variability measures using random forest methodology. Comput. Biol. Med. 70, 157-162. doi: 10.1016/j.compbiomed.2016.01.020 
Valderrama, C. E., Marzbanrad, F., Juarez, M., Hall-Clifford, R., Rohloff, P., and Clifford, G. D. (2020). Estimating birth weight from observed postnatal weights in a Guatemalan highland community. Physiol. Meas. 41:025008. doi: 10.1088/1361-6579/ab7350

Valderrama, C. E., Marzbanrad, F., Stroux, L., and Clifford, G. D. (2017). Templatebased quality assessment of the Doppler ultrasound signal for fetal monitoring. Front. Physiol. 8:511. doi: 10.3389/fphys.2017.00511

Valderrama, C. E., Marzbanrad, F., Stroux, L., Martinez, B., Hall-Clifford, R., Liu, C., et al. (2018a). "Improving the quality of point of care diagnostics with real-time machine learning in low literacy LMIC settings," in ACM SIGCAS Conference on Computing and Sustainable Societies (COMPASS 2018) (San Jose-Menlo Park, CA), Number 2, 1-11. doi: 10.1145/3209811.3209815

Valderrama, C. E., Stroux, L., Katebi, N., Paljug, E., Hall-Clifford, R., Rohloff, P., et al. (2019). An open source autocorrelation-based method for fetal heart rate estimation from one-dimensional Doppler ultrasound. Physiol. Meas. 40:025005. doi: 10.1088/1361-6579/ab033d

Valderrama, C. E., Stroux, L., Springer, D., and Clifford, G. D. (2018b). Open Source Code for Fetal Heart Estimator from One-dimensional Doppler Ultrasound Signals. doi: 10.5281/zenodo.1487995

Van Leeuwen, P., Geue, D., Lange, S., Hatzmann, W., and Grönemeyer, D. (2003). Changes in the frequency power spectrum of fetal heart rate in the course of pregnancy. Prenat. Diagn. 23, 909-916. doi: 10.1002/ pd.723

Van Leeuwen, P., Lange, S., Bettermann, H., Grönemeyer, D., and Hatzmann, W. (1999). Fetal heart rate variability and complexity in the course of pregnancy. Early Hum. Dev. 54, 259-269. doi: 10.1016/S0378-3782(98) 00102-9

Vest, A. N., Da Poian, G., Li, Q., Liu, C., Nemati, S., Shah, A. J., et al. (2018). An open source benchmarked toolbox for cardiovascular waveform and interval analysis. Physiol. Meas. 39:105004. doi: 10.1088/1361-6579/a ae021
Wakai, R. T. (2004). Assessment of fetal neurodevelopment via fetal magnetocardiography. Exp. Neurol. 190, 65-71. doi: 10.1016/j.expneurol.2004.04.019

Wallwitz, U., Schneider, U., Nowack, S., Feuker, J., Bauer, S., Rudolph, A., et al. (2012). Development of integrative autonomic nervous system function: an investigation based on time correlation in fetal heart rate patterns. J. Perinat. Med. 40, 659-667. doi: 10.1515/jpm-2012-0074

World Health Organization (2014). WHO Compendium of Innovative Health Technologies for Low-Resource Settings. Retrieved from: https://www.who.int/ medical_devices/innovation/compendium/en/ (accessed January 11, 2020).

World Health Organization (2016). WHO Recommendations on Antenatal Care for a Positive Pregnancy Experience. Retrieved from: https://www.who.int/ reproductivehealth/publications/maternal_perinatal_health/anc-positivepregnancy-experience/en/ (accessed October 11, 2019).

Wu, L., Hu, Y., Liu, X., Zhang, X., Chen, W., Alan, S., et al. (2018). Feature ranking in predictive models for hospital-acquired acute kidney injury. Sci. Rep. 8, 1-11. doi: 10.1038/s41598-018-35487-0

Zupan, J. (2005). Perinatal mortality in developing countries. N. Engl. J. Med. 352 2047-2048. doi: 10.1056/NEJMp058032

Conflict of Interest: The authors declare that the research was conducted in the absence of any commercial or financial relationships that could be construed as a potential conflict of interest.

Copyright (c) 2020 Valderrama, Marzbanrad, Hall-Clifford, Rohloff and Clifford. This is an open-access article distributed under the terms of the Creative Commons Attribution License (CC BY). The use, distribution or reproduction in other forums is permitted, provided the original author(s) and the copyright owner(s) are credited and that the original publication in this journal is cited, in accordance with accepted academic practice. No use, distribution or reproduction is permitted which does not comply with these terms. 